NBER WORKING PAPER SERIES

REAL OPTIONS WITH UNCERTAIN MATURITY AND COMPETITION

\author{
Kristian R. Miltersen \\ Eduardo S. Schwartz \\ Working Paper 12990 \\ http://www.nber.org/papers/w12990 \\ NATIONAL BUREAU OF ECONOMIC RESEARCH \\ 1050 Massachusetts Avenue \\ Cambridge, MA 02138 \\ March 2007
}

We thank Mark Garmaise, Jack Hughes and other seminar participants at UCLA for helpful comments. The views expressed herein are those of the author(s) and do not necessarily reflect the views of the National Bureau of Economic Research.

(C) 2007 by Kristian R. Miltersen and Eduardo S. Schwartz. All rights reserved. Short sections of text, not to exceed two paragraphs, may be quoted without explicit permission provided that full credit, including $\odot$ notice, is given to the source. 
Real Options With Uncertain Maturity and Competition

Kristian R. Miltersen and Eduardo S. Schwartz

NBER Working Paper No. 12990

March 2007

JEL No. G13,G31

\begin{abstract}
$\underline{\text { ABSTRACT }}$
We develop a new approach to dealing with real options problems with uncertain maturity. This type of situation is typical for $R \& D$ investments and mine or oil exploration projects. These types of projects are characterized by significant on-going investment costs until completion. Since time to completion is uncertain, the total investment costs will also be uncertain. Despite the fact that these projects include complicated American abandonment/switching options until completion and European options at completion (because of fixed final investment costs) we obtain simple closed form solutions. We apply the framework to situations in which the owner of the project has monopoly rights to the outcome of the project, and to situations in which there are two owners who simultaneously invest, but where only one of them may obtain the rights to the outcome. We expand the real options framework to incorporate game theoretic considerations, including a generalization of mixed strategies to continuous-time models in the form of abandonment intensities.
\end{abstract}

Kristian R. Miltersen

Department of Finance and Management Science

Helleveien 30

NO-5045 Bergen, NORWAY

kristian.r.miltersen@nhh.no

Eduardo S. Schwartz

Anderson Graduate School of Management

UCLA

110 Westwood Plaza

Los Angeles, CA 90095

and NBER

eduardo.schwartz@anderson.ucla.edu 


\title{
REAL OPTIONS WITH UNCERTAIN MATURITY AND COMPETITION
}

\author{
KRISTIAN R. MILTERSEN \\ Norwegian School of Economics and Business Administration \\ Department of Finance and Operations Research \\ Helleveien 30 \\ $N-5045$ Bergen \\ Norway
}

EDUARDO S. SCHWARTZ

Dept. of Finance (C4.13)

The John E. Anderson Graduate School of Management at UCLA

110 Westwood Plaza

Box 951481, UCLA

Los Angeles, CA 90095-1481

$U S A$

\begin{abstract}
We develop a new approach to dealing with real options problems with uncertain maturity. This type of situation is typical for R\&D investments and mine or oil exploration projects. These types of projects are characterized by significant on-going investment costs until completion. Since time to completion is uncertain, the total investment costs will also be uncertain. Despite the fact that these projects include complicated American abandonment/switching options until completion and European options at completion (because of fixed final investment costs) we obtain simple closed form solutions. We apply the framework to situations in which the owner of the project has monopoly rights to the outcome of the project, and to situations in which there are two owners who simultaneously invest, but where only one of them may obtain the rights to the outcome. We expand the real options framework to incorporate game theoretic considerations, including a generalization of mixed strategies to continuous-time models in the form of abandonment intensities.
\end{abstract}

\section{INTRODUCTION}

The valuation and analysis of investment projects in which time to completion is uncertain, such as R\&D investments and mine or oil exploration projects, is a very difficult task since there is uncertainty not only

E-mail addresses: kristian.r.miltersen@nhh.no, eduardo.schwartz@anderson.ucla.edu.

Date. September 2006. This version: March 13, 2007.

Journal of Economic Literature Classification. G31, G13.

Key words and phrases. Real options, uncertain finite maturity, competition.

We thank Mark Garmaise, Jack Hughes and other seminar participants at UCLA for helpful comments. Miltersen gratefully acknowledges financial support of Storebrand. Document typeset in $\mathrm{LAT}_{\mathrm{E}} \mathrm{X}$. 
about the outcome when the investment project is completed but also about the total investment costs. The problem gets even more complicated if the situation involves competitive interactions, such as the case when two different firms at the same time are competing to develop a pharmaceutical drug for a particular disease.

The real options approach has been applied to the valuation of $\mathrm{R} \& \mathrm{D}$ investment projects and to mine or oil exploitation projects. These type of projects involve not only uncertain cash flows after completion, but also uncertain costs and time to completion. So far in the literature, solutions to these type of problems have involved complex numerical solution techniques. Pindyck (1993) introduces a stochastic process to govern uncertain time to completion. Schwartz and Moon (2000) extend this approach to value an R\&D project with both uncertain costs and also uncertain value of the completed project. The solution involves a complex numerical procedure to solve elliptical PDEs. Schwartz (2004) solves the same R\&D investment problem using Monte Carlo simulation methods applying the Longstaff-Scwhartz procedure (cf. Longstaff and Schwartz, 2001) to value the American abandonment feature of the problem. Miltersen and Schwartz (2004) extend this framework to deal with competitive interactions and Hsu and Schwartz (2006) analyze the design of research incentives in $\mathrm{R} \& \mathrm{D}$ research. Both of the last two papers also apply Monte Carlo simulation solution techniques. One problem with the Monte Carlo numerical solution techniques used to solve these types of problems is that it cannot easily handle temporary suspensions of the projects. Hence, it is very difficult to compare investment projects with and without the temporary suspension option to isolate its value. Moreover, the numerical methods applied are designed to estimate the value of the projects, but they do not estimate the abandonment/swiching threshold levels very accurately.

In this paper we take a different approach. Without loosing the essential ingredients of the problem, we simplify the framework enough to get closed form solutions. Our simplified framework can be applied to many interesting and important cases of investments with uncertain time to completion. Moreover, our simplified framework allows us to look at cases not only where the owner of the investment project has exclusive rights to the outcome of the investment project if completed successfully, such as, e.g., an investment project where the owner of a property explores for gold, but also to look at cases where two (or more) firms are researching for the same product and at most one of them will be successful.

The main simplification we propose in our framework is that completion of the project is governed by an independent exponential distributed random variable. ${ }^{1}$ This implies that the value of the projects will be a solution to an ordinary differential equation, instead of a partial differential equation. Normally, the values of finite time option problems are solutions to partial differential equations, and since the type of investment projects we consider involve both American and European options, it is impossible to obtain closed form solutions without the simplification we propose. Carr (1998) uses a similar simplification as a numerical procedure to approach the value of financial American put options.

In our framework the owner of the investment project pays a certain amount of on-going investment costs per unit of time until a product or a mine is discovered. The completion date at which the product or the mine is discovered is uncertain. Since the completion date is uncertain, the on-going investment costs paid until completion will also be uncertain. At the completion date, when the product or mine is discovered, the owner of the investment project has to decide whether it is worth while to make a final investment in building a plant to produce the product or extract the mineral. To make this decision the owner of the investment project compares the final investment costs with the present value of the net cash flows that could

\footnotetext{
${ }^{1}$ This implies that the conditional probability of completion per unit of time is constant. This assumption might be somewhat unrealistic for some situations.
} 
be generated by selling the product or mineral. This present value of the net cash flows we will call the value of the outcome of the investment project. We assume that the value of the outcome evolves stochastically through time and that it can be observed or estimated by the owner of the project at any point in time. The idea here is that as investment and/or time progresses, the owner of the investment project changes his estimate of the present value of the net cash flows that could be derived from the investment project and that this updated information may influence the owner's willingness to continue with the investment project.

Within this framework we analyze two general types of models. In the first type of models, monopoly models, the owner of the project has exclusive rights to (the value of) the outcome of the investment project if and when it is completed. In the second type of models, duopoly models, two owners of similar investment projects compete to be the first to complete their investment projects because only the first to complete will get the right to (the value of) the outcome.

We analyze three different monopoly models. In the first model we allow the owner of the investment project to abandon the investment project if the future prospects are not good enough. Since the owner of the investment project is paying on-going investment costs to keep the investment project alive, for low enough values of the outcome it will be optimal to abandon the investment project.

In the second model we allow the owner of the investment project to switch from an active investment project into a passive investment project and vise-versa. When the investment project is active the owner incurs on-going investment costs and there is a positive probability of completing the project. When the investment project is passive there are no on-going investment costs but also no possibility of completion. If the value of the outcome is high enough it will be worth while to pay the on-going investment costs to have the possibility of completion. In the passive state there are no on-going investment costs, so if the value of the outcome is low enough, the owner would like to switch to the passive state. Hence, there will be a threshold value of the outcome above which it is optimal to have the investment project active and below which it is optimal to have it passive. Moreover, it will never be optimal to abandon the investment project, since it is costless to keep it passive.

Finally, we consider a model with both switching and abandonment options. In this model the owner can switch between two different levels of investment activity, high and low. At the high investment activity level, the owner pays high on-going investment costs and the probability of completion per unit of time is high. That is, there is a high chance of discovering a product or a mine per unit of time. At the low investment activity level, the on-going investment costs are lower but the probability of completion per unit of time is also lower. In this setting there will be a threshold value of the outcome above which it is optimal to have a high investment activity level and below which it is optimal to have a low investment activity level. Moreover, there will be another threshold value of the outcome below which it is optimal to abandon the investment project.

In all these three models the owner of the investment project has monopoly rights to the value of the outcome, that is, there is no competition as to whom owns the right to the value of the outcome when the investment project is eventually completed. We then look at another type of models where there are two different owners of investment projects, but only the one whose investment project is completed first gets the value of the outcome. Here the values of the investment projects and the decisions to abandon or switch will depend not only on the value of the outcome but also on the competitive interactions between the two owners (players). To be able to solve these types of problems we need to expand the real options framework 
we have developed to incorporate game theoretic considerations, where the optimal actions of each player depend on the (optimal) actions of the other player. To simplify the analysis we assume that the two players are identical with respect to the size of their investment costs and their probability of completion.

We again analyze models with abandonment and with switching. In order to analyze the abandonment option we first consider the case where one of the players (the leader) has a un-modeled advantage over the other player (the follower), such as slightly less investment costs or more financial resources available. In this model the two players have different abandonment threshold levels. We then look at the model where the two players are truly identical and we analyze the game that occurs. We obtain three (Nash) equilibria. One of these equilibria involves a strategy for each of the two players where they each chooses an abandonment intensity. The abandonment intensity gives for each player the probability of abandoning their individual investment projects at each instant of time. This equilibrium concept is a natural extension of mixed strategy equilibria to continuous-time models and as far as we are aware it has not appeared in the literature.

We finally analyze the duopoly model with the switching option between a passive and an active state. In this case the switching threshold levels for the two players are again determined as a Nash equilibrium in a game between the two players.

The simplified framework we develop allows us to obtain simple closed form solutions to the values of the investment projects and the threshold levels ${ }^{2}$ of all the models described above. We are therefore able to easily perform comparative statics of each model and compare the different models. We hope that this simple and flexible framework will generate more insight and more analysis and solutions of many interesting real options problems.

The monopoly models are presented in Section 2 and the duopoly models in Section 3. Section 4 develops some extensions of the models and Section 5 concludes. To simplify the presentation some of the derivations are given in the Appendix.

\section{Monopoly Models}

In this section we consider models in which the owner of the investment project does not face any competition as to whom will have the right to the value of the outcome at completion of the investment project. In that sense the owner has a monopoly right to the value of the outcome of the investment project.

Consider an investment project that requires on-going investment costs at the rate of $k$ per unit of time. The investment project will be completed at a random date $\tau$ at which date the owner of the investment project has the option to pay final (fixed) investment costs $K$ in order to obtain the (net present) value of the outcome $V$. In this setup the value of the investment project at the random completion date, $\tau$, would be $\max \left\{V_{\tau}-K, 0\right\}$.

For the case of an R\&D investment project, $k$ would represent the rate of expenses for the R\&D program, $\tau$ would represent the random date at which the research project is completed, $K$ would represent the costs of building a plant for manufacturing the products obtained from the research project, and $V$ would represent the net present value of the benefits from selling the products.

Similarly, for the case of a mine or an oil exploration project, $k$ would represent the rate of expenses for the exploration phase, $\tau$ would represent the random date at which a natural resource may be discovered,

\footnotetext{
${ }^{2}$ In the combined abandonment and switching model the two threshold levels have to be found numerically, but a very simple goal-seek in, e.g., Excel finds these two values in less than a tenth of a second.
} 
$K$ would represent the costs of developing the mine or the oil field, and $V$ would represent the net present value of the benefits from exploiting the natural resource discovered.

We assume that the value of the outcome, $V$, can be observed (estimated) at any date $t$ by the owner of the investment project. This value we denote $V_{t}$. We assume that the dynamics of $V$ is given by the geometric Brownian motion

$$
d V_{t}=V_{t} \mu d t+V_{t} \sigma d W_{t}
$$

Here $\sigma$ is the instantaneous volatility of the value process, $\mu$ is its instantaneous drift, and $W$ represents a Brownian motion.

We assume that the random time to completion, $\tau$, is exponentially distributed with intensity, $\lambda$, and that the time to completion is independent of the value process, $\left\{V_{t}\right\}_{t \in[0, \infty)}$. Hence, the expected time to completion is $T=\frac{1}{\lambda}$ without taking into account any abandonment possibilities nor any possibilities of switching to different levels of intensity, $\lambda$. Finally, we assume that the riskless interest rate, $r$, is constant. In order to avoid the possibility of infinite values of the investment projects which we consider in this paper, we assume that the instantaneous drift of the value process, $\mu$, is strictly less than the riskless rate, $r$, i.e. $\mu<r$. The memorylessness feature of the exponential distribution implies that the expected time (and the whole distribution of the time) to completion of the investment project does not dependent on calendar time. Hence, time to completion and the completion date will have the same distribution, so we will not distinguish between these two terms. Moreover, the value of the investment project at a given date, $t$, depends only on the value of the outcome, $V_{t}$, and not on the calendar date $t$ itself. This property considerably simplifies the analysis and allows us to obtain closed form solutions for many important and interesting cases.

2.1. Model with Abandonment Option. We first consider the model with abandonment. In addition to the option to the value of the outcome at the completion date, $\tau$, the owner has the option to abandon the investment project at any date before completion. This means that the owner would loose the option to the value of the outcome but on the other hand she/he will save the remaining (future) on-going investment costs. Given that the owner of the investment project is spending on-going investment costs at the rate $k$ per unit of time there will be a threshold level, $A_{M}$, so that if the value of the outcome, $V$, drops below this threshold level at a certain date, then it will be optimal to abandon the investment project at that date. We denote the value of the investment project $M(V) .{ }^{3}$ The investment project is a contingent claim on the value of the outcome, $V$. Applying standard arguments, $M(V)$ must satisfy the system of ODEs ${ }^{4}$

$$
\frac{1}{2} \sigma^{2} V^{2} M^{\prime \prime}(V)+\mu V M^{\prime}(V)-(r+\lambda) M(V)-k=0 \quad \text { when } A_{M}<V<K
$$

\footnotetext{
${ }^{3}$ For notational simplicity and clarity we write the value of the investment project as a function of current value of the outcome instead of the date $t$ value of the dynamic value process $V_{t}$.

${ }^{4}$ Either we can assume that there exists a sufficiently dynamically complete financial market and that we have modeled the dynamics of the value process, $V$ in Equation (1), under a risk neutral measure, or we can assume that the owner assesses the value of the investment project at date $t$ as

$$
M\left(V_{t}\right)=\max _{\nu \in \mathcal{S}} E_{t}\left[1_{\{\tau \leq \nu\}} e^{-r(\tau-t)} \max \left\{V_{\tau}-K, 0\right\}-\int_{t}^{\min \{\tau, \nu\}} k e^{-r(s-t)} d s\right] .
$$

Here $\nu$ represents the stopping time for abandonment which the owner chooses to follow. $E_{t}$ denotes expectations given all information at date $t$ and $1_{\{\tau \leq \nu\}}$ denotes the indicator function that the stopping time for completion, $\tau$, occurs before the stopping time for abandonment, $\nu$. The owner maximizes her/his value of expected future cash flows by choosing the optimal abandonment stopping time, $\nu$, from the set, $\mathcal{S}$, of all optional stopping times. The assessment in Equation (2) reflects a risk neutral owner.
} 
and

$$
\frac{1}{2} \sigma^{2} V^{2} M^{\prime \prime}(V)+\mu V M^{\prime}(V)-(r+\lambda) M(V)-k+\lambda(V-K)=0 \quad \text { when } K<V .
$$

Here we have implicitly assumed that the abandonment threshold level, $A_{M}$, is below the final investment $\operatorname{costs} K$ (this $K$ is also the exercise price of the option to the value of the outcome). ${ }^{5}$ Equation (3a) describes the value of the investment project when the value of the outcome, $V$, is below the exercise price, $K$, that is, if the option to the value of the outcome is out of the money (should the investment project be completed immediately). For the rest of the paper we will say that the investment project is out of the money to describe this situation. (Obviously, to describe the opposite situation, namely that $V \geq K$, we say that the investment project is in the money.) Equation (3a) reflects the fact that with intensity $\lambda$ (i.e., with probability $\lambda$ per unit of time) the investment project would be completed and because the investment project is out of the money, the value of the investment project will jump down to zero. This corresponds to a loss in value of $M(V) .{ }^{6}$ In addition, the owner has to pay on-going investment costs at the rate $k$ per unit of time in order to keep the investment project alive. Equation $(3 \mathrm{~b})$ describes the value of the investment project when it is in the money. This equation reflects the fact that with intensity $\lambda$ the value of the investment project would jump to the completion value, $V-K$. This corresponds to a jump in value of $V-K-M(V)$. In addition, the owner still has to pay on-going investment costs at the rate $k$ per unit of time in order to keep the investment project alive. We can solve each of the two ODEs, (3a) and (3b), separately for given boundary conditions. We denote the general solution for $\operatorname{ODE}(3 \mathrm{a}) M_{1}(V)$ and the general solution for ODE $(3 \mathrm{~b}) M_{2}(V)$. The boundary conditions will be used to tie the different general solutions for the different areas of the value of the outcome, $V$, together into one continuous (and differentiable) value function for the investment project for all values of the outcome, $V$. The general solutions to each of these two ODEs, (3a) and (3b), are

$$
M_{1}(V)=m_{11} V^{y_{1}}+m_{12} V^{y_{2}}-\frac{k}{r+\lambda} \quad \text { when } A_{M} \leq V \leq K
$$

and

$$
M_{2}(V)=m_{21} V^{y_{1}}+m_{22} V^{y_{2}}+\frac{\lambda V}{r+\lambda-\mu}-\frac{k+\lambda K}{r+\lambda} \quad \text { when } K \leq V .
$$

Here the powers, $y_{1}$ and $y_{2}$, are given by

$$
y_{1}=\frac{\left(\frac{1}{2} \sigma^{2}-\mu\right)+\sqrt{\left(\mu-\frac{1}{2} \sigma^{2}\right)^{2}+2(r+\lambda) \sigma^{2}}}{\sigma^{2}}>1
$$

and

$$
y_{2}=\frac{\left(\frac{1}{2} \sigma^{2}-\mu\right)-\sqrt{\left(\mu-\frac{1}{2} \sigma^{2}\right)^{2}+2(r+\lambda) \sigma^{2}}}{\sigma^{2}}<0
$$

\footnotetext{
${ }^{5}$ We analyze the (much simpler) situation when $A_{M}$ is above $K$ in Appendix A.

${ }^{6}$ One may argue that it is unrealistic to assume that the investment project is valueless if it is completed at a time when the value of the outcome, $V$, is below the fixed investment costs, $K$. The owner would still be able to claim the right to her/his discovery (either by patenting or other legal actions) and eventually put it into production at a later date. That is, a more realistic assumption would be that at the date of completion of the investment project the owner receives a perpetual American call option on the value of the outcome with exercise price equal to the final investment costs, $K$. We analyze this extension of the model in Section 4.
} 
Note that $y_{1}>1$ and that $y_{2}<0$. Since the value of the investment project can never exceed the value of the outcome, $V$, we know that $\lim _{V \rightarrow \infty} \frac{M_{2}(V)}{V}$ must be finite (in fact, it must be less than one). This implies that $m_{21}=0$ since $y_{1}>1$. Hence, we can eliminate the $V^{y_{1}}$ term in Equation (4b). Moreover, we can substitute $T=\frac{1}{\lambda}$ for the expected (remaining) time to completion of the investment project to get

$$
M_{1}(V)=m_{11} V^{y_{1}}+m_{12} V^{y_{2}}-\frac{k T}{1+r T} \quad \text { when } A_{M} \leq V \leq K
$$

and

$$
M_{2}(V)=m_{22} V^{y_{2}}+\frac{V}{1+(r-\mu) T}-\frac{k T+K}{1+r T} \quad \text { when } K \leq V .
$$

The value of the investment project has the following boundary conditions

$$
\begin{aligned}
M_{1}\left(A_{M}\right) & =0, \\
M_{1}^{\prime}\left(A_{M}\right) & =0, \\
M_{1}(K) & =M_{2}(K),
\end{aligned}
$$

and

$$
M_{1}^{\prime}(K)=M_{2}^{\prime}(K)
$$

Condition (7a) reflects the fact that at the abandonment threshold level, $A_{M}$, the value of the investment project is zero. Condition (7b) is the usual smooth pasting condition at the abandonment threshold level. Condition(s) (7c) (and (7d)) reflect(s) the fact that the value function should be continuous (and differentiable) at the point when the two differential equations (3a) and (3b) meet at the final investment costs, $K$. We can solve the system of equations (7) in the four unknowns, $m_{11}, m_{12}, m_{22}$, and $A_{M}$. The solution is

$$
\begin{aligned}
m_{11} & =\frac{\left(1+\left(r-y_{2} \mu\right) T\right) K^{1-y_{1}}}{\left(y_{1}-y_{2}\right)(1+(r-\mu) T)(1+r T)}, \\
A_{M} & =\left(\frac{y_{2} k T}{\left(y_{2}-y_{1}\right)(1+r T) m_{11}}\right)^{\frac{1}{y_{1}}}, \\
m_{12} & =-\frac{y_{1} m_{11} A_{M}^{y_{1}-y_{2}}}{y_{2}},
\end{aligned}
$$

and

$$
m_{22}=m_{12}+\frac{\left(1+\left(r-y_{1} \mu\right) T\right) K^{1-y_{2}}}{\left(y_{1}-y_{2}\right)(1+(r-\mu) T)(1+r T)} .
$$

Finally, the value of the investment project is

$$
M(V)= \begin{cases}0 & \text { when } V<A_{M}, \\ m_{11} V^{y_{1}}+m_{12} V^{y_{2}}-\frac{k T}{1+r T} & \text { when } A_{M} \leq V<K, \\ m_{22} V^{y_{2}}+\frac{V}{1+(r-\mu) T}-\frac{k T+K}{1+r T} & \text { when } K \leq V .\end{cases}
$$

We made an implicit assumption when setting up the system of ODEs (3) above, namely that the abandonment threshold level, $A_{M}$, is below the final investment costs, $K$. If it turns out that by solving the system of equations (7), the solution to $A_{M}$ from Equation (8b) is greater than $K$, we must reconsider the whole 


\begin{tabular}{lrr}
\hline \hline \multicolumn{3}{l}{ Parameters for the dynamics of the value process, $V$} \\
Instantaneous drift & $\mu$ & $3 \%$ per year \\
Instantaneous volatility & $\sigma$ & $40 \%$ per year \\
\hline \multicolumn{4}{c}{ Parameters for the investment project } \\
Expected time to completion & $T$ & 5 years \\
On-going investment costs rate & $k$ & $\$ 1$ per year \\
Final (fixed) investment costs & $K$ & $\$ 5$ \\
\hline \multicolumn{4}{c}{ Other parameters } \\
Riskless interest rate & $r$ & $5 \%$ per year \\
\hline \hline
\end{tabular}

TABle 1. Parameter values for numerical illustration: the base case.

problem, because in this situation the investment project is always in the money when it is kept alive. We analyze this situation, which turns out to be much simpler, in Appendix A. In this situation the value of the investment project is

$$
M(V)= \begin{cases}0 & \text { when } V<\tilde{A}_{M}, \\ m_{32} V^{y_{2}}+\frac{V}{1+(r-\mu) T}-\frac{k T+K}{1+r T} & \text { when } \tilde{A}_{M} \leq V .\end{cases}
$$

Here $\tilde{A}_{M}$ and $m_{32}$ are

$$
\tilde{A}_{M}=\frac{y_{2}(1+(r-\mu) T)(k T+K)}{\left(y_{2}-1\right)(1+r T)}
$$

and

$$
m_{32}=-\frac{\tilde{A}_{M}^{1-y_{2}}}{y_{2}(1+(r-\mu) T)} .
$$

Note that the abandonment threshold level in this situation (denoted $\tilde{A}_{M}$ ) is, in general, different from $A_{M}$ from Equation (8b). For notational simplicity, however, we will always refer to $A_{M}$ as the abandonment threshold level for the rest of the paper even though strictly speaking we should distinguish between $A_{M}$ and $\tilde{A}_{M}$ depending on whether $A_{M}<K$ or $\tilde{A}_{M} \geq K$. These two events are mutually exclusively and collectively exhaustive. $^{7}$

To illustrate the nature of the solution, we consider an investment project that requires on-going investment costs of $\$ 1$ (million) per year until completion. We assume that the expected time to completion is 5 years and that at completion the investment project requires final investments of $\$ 5$ (million) in order to obtain the cash flows of the outcome. The instantaneous drift of the value process is $3 \%$ and its instantaneous volatility is $40 \%$ per year. Finally, the interest rate is $5 \%$ per year. The parameters of this numerical illustration, which we call our base case, is summarized in Table 1.

\footnotetext{
${ }^{7}$ Thorough extensive numerical investigations have confirmed that in the parameter space, $\left\{(\mu, \sigma, r, \lambda, k, K) \in \mathbb{R}^{6} \mid \mu<r, \sigma>\right.$ $0, r>0, \lambda>0, k \geq 0, K \geq 0\}$, the sets $\left\{A_{M} \geq K\right\}$ and $\left\{\tilde{A}_{M} \geq K\right\}$ are (generically) identical. This is also the case for the sets $\left\{A_{M}>K\right\}$ and $\left\{\tilde{A}_{M}>K\right\}$ and also $\left\{A_{M}=K\right\}$ and $\left\{\tilde{A}_{M}=K\right\}$. However, we have not formally proven this result analytically.
} 


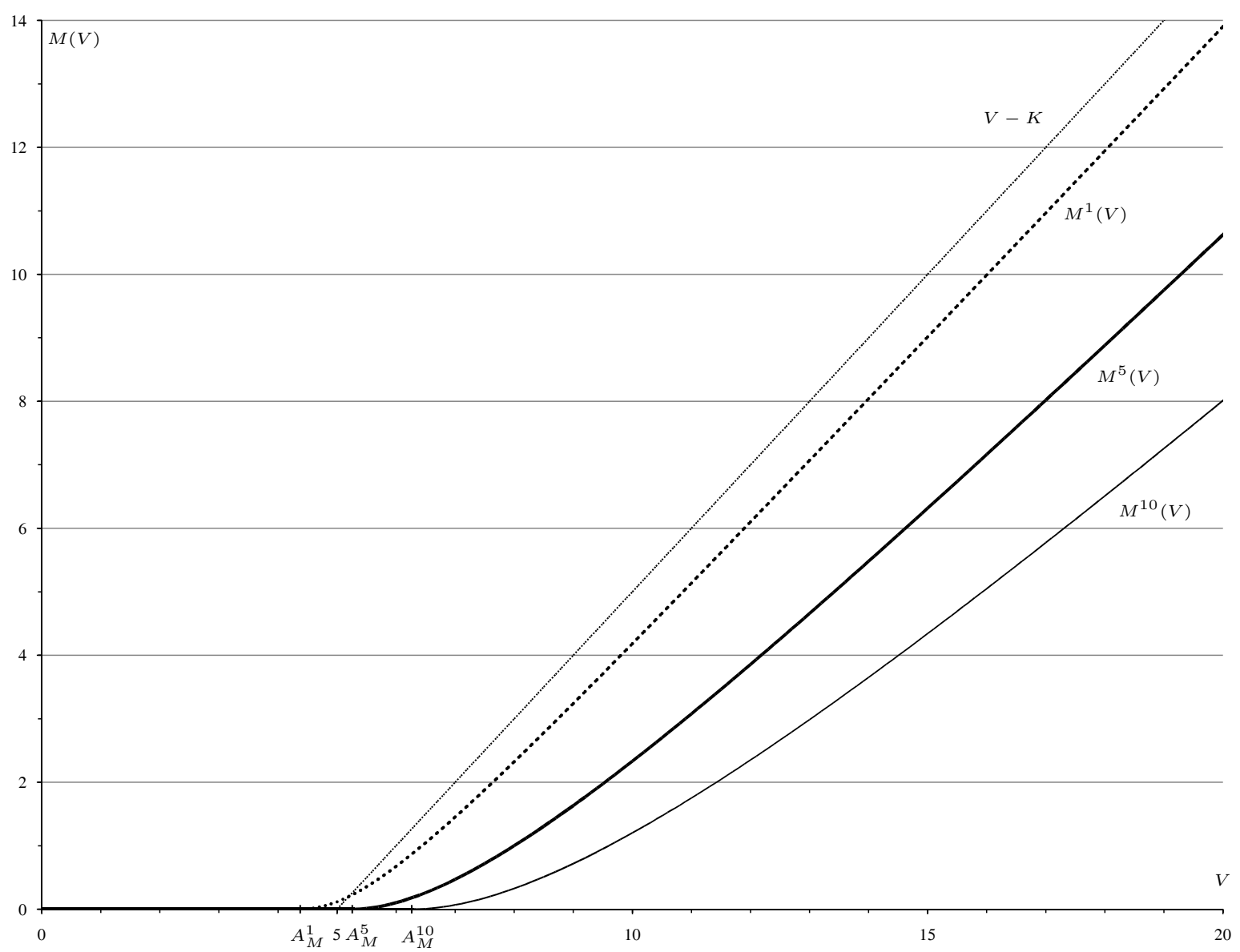

Figure 1. Values of investment projects (denoted $M^{1}(V), M^{5}(V)$, and $M^{10}(V)$ ) as a function of the value of the outcome, $V$, for three different values of expected time to completion, $T=1, T=5$, and $T=10$ years. The figure also shows the corresponding abandonment threshold levels (denoted $A_{M}^{1}, A_{M}^{5}$, and $A_{M}^{10}$ ) on the horizontal axis for the three different values of expected time to completion. The exact levels are $A_{M}^{1}=4.37$, $A_{M}^{5}=5.26$, and $A_{M}^{10}=6.26$. Finally, the figure also shows the completion values of the investment project (denoted $V-K$ ) as a function of the value of the outcome. All other parameters are given in Table 1.

In Figure 1 we show the values of the investment projects as a function of the value of the outcome, $V$, for three different values for the expected time to completion, $T=1, T=5$, and $T=10$ years. We denote the corresponding values of the investment projects as $M^{1}(V), M^{5}(V)$, and $M^{10}(V)$. As can be seen from the figure the values of the investment projects are higher when the expected time to completion is shorter. Since the owner of the investment project pays on-going investment costs in the order of $\$ 1$ per unit of time the investment project is more valuable with shorter expected time to completion. For realistic values of on-going investment costs the typical situation is that the value of the investment project decreases with longer expected time to completion. However, for very low values of on-going investment costs rate, $k$, (say $k$ is in the order of $1-4 \%$ of the value of the final investment costs, $K$ ) the value of the investment project may be increasing in the expected time to completion. This is so because the value increasing effect of 
postponing the final investment costs, $K$, at completion may dominate the value decreasing effect of paying the on-going investment costs for a longer time. ${ }^{8}$ Hence, in general, the value of the investment project may be non-monotonic in the expected time to completion.

In Figure 1 we also show the corresponding abandonment threshold levels on the horizontal axis for the three different values of the expected time to completion, denoted $A_{M}^{1}, A_{M}^{5}$, and $A_{M}^{10}$. When the expected time to completion is 5 years or 10 years the corresponding abandonment threshold levels are above the final investment costs, $K$, and are therefore derived from Equation (11a). The exact levels are $A_{M}^{5}=5.26$ and $A_{M}^{10}=6.26$. When the expected time to completion is 1 year the corresponding abandonment threshold level is below the final investment costs, $K$, and it is therefore derived from Equation (8b). The exact level is $A_{M}^{1}=4.37$.

Finally, in Figure 1 we also show the completion values of the investment projects as function of the value of the outcome. We denote these values $V-K$. In most cases the value of the investment projects is below the corresponding completion values mainly because the owner has to pay the on-going investment cost in order to keep the investment projects alive. However, for investment projects that are out of the money or at least not too far in the money, (early) completion can result in a negative jump in value, because the value of the project (before completion) includes the chance of a higher completion value.

When the abandonment threshold level is above the final investment costs, $K$, an increase in the expected time to completion of the investment project will typically lead to an even higher abandonment threshold level since the effect that the owner has to pay the on-going investment costs for a longer time typically dominates. However, when the abandonment threshold level is below the final investment costs, $K$, an increase in the expected time to completion of the investment project may lead to a lower abandonment threshold level since the effect that a longer expected time to completion increases the likelihood that the investment project will be completed in the money dominates the effect that the owner has to pay the on-going investment costs for a longer time. In Figure 2 we show the abandonment threshold levels as a function of expected time to completion, $T$, with our base case parameters. As we have just explained, typically, the abandonment threshold levels will be an increasing function of the expected time to completion. However, for short expected time to completion (for $T$ below 0.36 years with our base case parameters), the abandonment threshold levels decrease with increased expected time to completion. Hence, even with our base case parameters, Figure 2 demonstrates that the abandonment threshold levels may be a non-monotonic function of the expected time to completion. For $T=0$, the abandonment threshold level is clearly equal to the final investment costs, $K=5$. Finally, note that the abandonment threshold level function in Figure 2 is smooth around the level 5 on the vertical axis where the abandonment threshold level shifts from being defined by Equation (8b) to being defined by Equation (11a), as claimed in footnote 7 .

2.2. Model with Switching Option. Now consider the same investment project as in sub-section 2.1 but with the following modification. The owner of the investment project can temporarily suspend investing in the project. When the investment project is suspended both the on-going investment costs rate, $k$, and the intensity of completion, $\lambda$, switch to zero. In other words, the owner has the option to switch from an active

\footnotetext{
${ }^{8}$ In general this effect may again be dominated by another value decreasing effect. That is, if the value of the outcome, $V$, is high enough compared to the final investment costs, $K$, then the value decreasing effect of having to wait a longer time to get the value of the outcome may dominate the value increasing effect of postponing of the final investment costs. This effect is caused by our assumption that the instantaneous drift, $\mu$, of the value process is less than the riskless interest rate, $r$. Moreover, the greater the difference between $r$ and $\mu$, the stronger is this effect.
} 




Figure 2. Abandonment threshold levels (denoted $A_{M}$ ) as a function of expected time to completion, $T$. All other parameters are given in Table 1.

investment project to a passive (mothballed) investment project and vice versa costlessly. Clearly, in this model it would never be optimal to abandon the investment project since there are no costs of keeping the investment project passive. Abandonment is a non-reversible decision, whereas switching allows for multiple changes of state. Since when the investment project is passive there is no possibility of completion, there will be a threshold level, $S_{N}$, so that if the value of the outcome, $V$, is above this threshold level at a certain date, then it would be optimal to be in the active state at that date, and if $V$ is below this threshold level it would be optimal to be in the passive state at that date. We call $S_{N}$ the switching point. It is easy to see that $S_{N}$ will be above the final investment costs, $K$, since by mothballing the investment project when it is out of the money, the owner can avoid that the investment project is ever completed out of the money. Let $N(V)$ denote the value of the investment project for this model. Following arguments similar to the previous model, $N(V)$ must satisfy the system of ODEs

$$
\frac{1}{2} \sigma^{2} V^{2} N^{\prime \prime}(V)+\mu V N^{\prime}(V)-r N(V)=0 \quad \text { when } V<S_{N}
$$

and

$$
\frac{1}{2} \sigma^{2} V^{2} N^{\prime \prime}(V)+\mu V N^{\prime}(V)-(r+\lambda) N(V)-k+\lambda(V-K)=0 \quad \text { when } S_{N}<V .
$$


Equation (12a) describes the value of the investment project when it is passive. This equation reflects the fact that there is no chance (or risk) that the project will be completed as long as it is passive, i.e. $\lambda=0$, and that there are no on-going investment costs when the investment project is passive, i.e. $k=0$. Equation (12b) describes the value of the investment project when it is active. This equation has the same terms as Equation (3b) because, as we have argued, the investment project is always in the money when it is active. The general solutions to each of the two ODEs, (12a) and (12b), which we denote $N_{1}(V)$ and $N_{2}(V)$, are

$$
N_{1}(V)=n_{11} V^{x_{1}}+n_{12} V^{x_{2}} \quad \text { when } V \leq S_{N}
$$

and

$$
N_{2}(V)=n_{21} V^{y_{1}}+n_{22} V^{y_{2}}+\frac{V}{1+(r-\mu) T}-\frac{k T+K}{1+r T} \quad \text { when } S_{N} \leq V .
$$

Here the powers, $x_{1}$ and $x_{2}$, are given by

$$
x_{1}=\frac{\left(\frac{1}{2} \sigma^{2}-\mu\right)+\sqrt{\left(\mu-\frac{1}{2} \sigma^{2}\right)^{2}+2 r \sigma^{2}}}{\sigma^{2}}>1
$$

and

$$
x_{2}=\frac{\left(\frac{1}{2} \sigma^{2}-\mu\right)-\sqrt{\left(\mu-\frac{1}{2} \sigma^{2}\right)^{2}+2 r \sigma^{2}}}{\sigma^{2}}<0 .
$$

The powers $y_{1}$ and $y_{2}$ are still defined by the system of equations (5). Comparing the powers $x_{1}$ and $x_{2}$ with the corresponding powers $y_{1}$ and $y_{2}$, we see that $y_{1}>x_{1}>1$ and that $y_{2}<x_{2}<0$. In our general solution in Equation (13a) we can eliminate the $V^{x_{2}}$ term, since the value of the investment project must be zero when the value of the outcome, $V$, converge to zero. Since $x_{2}<0$, the coefficient in front of the $V^{x_{2}}$ term must be zero in order for the value of the investment project not to explode as the value of the outcome, $V$, converge to zero. In addition, using the same arguments as in sub-section 2.1, we know that $\lim _{V \rightarrow \infty} \frac{N_{2}(V)}{V}$ must be finite. Hence, we can eliminate the $V^{y_{1}}$ term in Equation (13b). The value of the investment project has the following boundary conditions

$$
N_{1}\left(S_{N}\right)=N_{2}\left(S_{N}\right)
$$

and

$$
N_{1}^{\prime}\left(S_{N}\right)=N_{2}^{\prime}\left(S_{N}\right)
$$

The two conditions, (15a) and (15b), reflect the fact that the value function should be continuous and differentiable at the point when the two differential equations (12a) and (12b) meet at the switching point, $S_{N}$. In order to find the optimal switching point itself, $S_{N}$, we solve

$$
\lambda\left(S_{N}-K\right)=\lambda N_{2}\left(S_{N}\right)+k .
$$

Equation (15c) is based on the following instantaneous trade-off argument. At each instant in time the owner considers the instantaneous costs and benefits from switching between an active and a passive investment project. The increased instantaneous benefits from switching from a passive state to an active state is the 
increased intensity of completion which has a value flow $\lambda(V-K)$ per unit of time. On the other hand the increased instantaneous costs of switching are (i) the increased intensity of loosing the investment project (an inevitable consequence of completion) which has a value flow $\lambda N_{2}(V)$ per unit of time ${ }^{9}$ and (ii) the increased on-going investment costs at the rate $k$ per unit of time.

Note that Equation (15c) also implies that the value function, $N(V)$, will be two times differentiable at the switching point, $S_{N}$. To see this note that $N_{2}$ is a solution to the ODE (12b), hence,

$$
\frac{1}{2} \sigma^{2} S_{N}^{2} N_{2}^{\prime \prime}\left(S_{N}\right)=r N_{2}\left(S_{N}\right)-\mu S_{N} N_{2}^{\prime}\left(S_{N}\right)+\lambda N_{2}\left(S_{N}\right)+k-\lambda\left(S_{N}-K\right) .
$$

Using Equation (15c), we can eliminate the last terms in Equation (16), so we have

$$
\frac{1}{2} \sigma^{2} S_{N}^{2} N_{2}^{\prime \prime}\left(S_{N}\right)=r N_{2}\left(S_{N}\right)-\mu S_{N} N_{2}^{\prime}\left(S_{N}\right) .
$$

On the other hand $N_{1}$ is a solution to the ODE (12a), hence,

$$
\frac{1}{2} \sigma^{2} S_{N}^{2} N_{1}^{\prime \prime}\left(S_{N}\right)=r N_{1}\left(S_{N}\right)-\mu S_{N} N_{1}^{\prime}\left(S_{N}\right) .
$$

Using Equations (15a) and (15b) we conclude that the right hand sides of Equations (17) and (18) are identical, hence the left hand sides must also be identical. Since $S_{N}>0$ and $\sigma>0$, we conclude that

$$
N_{1}^{\prime \prime}\left(S_{N}\right)=N_{2}^{\prime \prime}\left(S_{N}\right) \text {. }
$$

In order to find the value of the investment project, we solve the system of equations (15) in the three unknowns, $n_{11}, n_{22}$, and $S_{N}$, to get

$$
\begin{aligned}
S_{N} & =\frac{\left(x_{1}+\left(x_{1}-y_{2}\right) r T\right)(1+(r-\mu) T)(k T+K)}{\left(x_{1}-1+\left(x_{1}-y_{2}\right)(r-\mu) T\right)(1+r T)}, \\
n_{11} & =\frac{y_{2}(1+(r-\mu) T)(k T+K)+\left(1-y_{2}\right)(1+r T) S_{N}}{\left(x_{1}-y_{2}\right)(1+(r-\mu) T)(1+r T) S_{N}^{x_{1}}},
\end{aligned}
$$

and

$$
n_{22}=\frac{x_{1}(1+(r-\mu) T)(k T+K)-\left(x_{1}-1\right)(1+r T) S_{N}}{\left(x_{1}-y_{2}\right)(1+(r-\mu) T)(1+r T) S_{N}^{y_{2}}} .
$$

From Equation (15c) it is clear that $S_{N}>k T+K$. To see this note that $k T+K$ would have been the optimal switching point if the owner did not take into account the costs of losing the investment project. That is, if the term $\lambda N_{2}\left(S_{N}\right)$ would not have been present on the right hand side of Equation (15c) then the optimal switching point would have been $k T+K$. Since $N_{2}\left(S_{N}\right)$ is strictly positive, the optimal switching point must be higher than $k T+K$. Finally, the value of the investment project is

$$
N(V)= \begin{cases}n_{11} V^{x_{1}} & \text { when } V<S_{N}, \\ n_{22} V^{y_{2}}+\frac{V}{1+(r-\mu) T}-\frac{k T+K}{1+r T} & \text { when } S_{N} \leq V .\end{cases}
$$

The solution to the switching point from Equation (20a), which essentially is based on the instantaneous trade-off argument from Equation (15c), also has the property that it maximizes the value function, $N(V)$, globally. That is, if we consider the value of the investment project, now denoted $N(V ; S)$, not only as a function of the value of the outcome, $V$, but also as the function of a given pre-committed switching point,

\footnotetext{
${ }^{9}$ Note that it does not matter whether we use $N_{1}\left(S_{N}\right)$ or $N_{2}\left(S_{N}\right)$ for the value of the investment project at the switching point since by Equation (15a) the two functions have the same value at the switching point, $S_{N}$.
} 
$S$, then the switching point solution, $S_{N}$, from Equation (20a), maximizes the function $S \mapsto N(V ; S)$ for any choice of $V$.

Note that this switching option model has the standard perpetual American call option as a limit case. That is, if $\lambda=\infty$, switching to an active investment project will trigger immediate completion. This corresponds to the decision of exercising a perpetual American call option written on the value of the outcome, $V$, and with the final (fixed) investment costs, $K$, as exercise price. Inserting $T=0$ (corresponding to $\lambda=\infty$ ) into Equation (20a) gives the well-known optimal exercise threshold level ${ }^{10}$

$$
S_{N}=\frac{x_{1}}{\left(x_{1}-1\right)} K
$$

and the well-known value

$$
N(V)= \begin{cases}\frac{V}{x_{1}}\left(\frac{V}{S_{N}}\right)^{x_{1}-1} & \text { when } V<S_{N}, \\ V-K & \text { when } S_{N} \leq V\end{cases}
$$

In Figure 3 we show the values of the investment projects as a function of the value of the outcome, $V$, for three different values of the expected time to completion, $T=1, T=5$, and $T=10$ years. We denote the corresponding values of the investment projects as $N^{1}(V), N^{5}(V)$, and $N^{10}(V)$. As can be seen from the figure, we have the same observation as for the model with the abandonment option, namely that the values of the investment projects are higher when the expected time to completion is shorter. Now, since there are no costs of keeping the investment projects passive, the projects have strictly positive values even for very low values of the outcome. For comparison we also show in Figure 3 the value of the corresponding investment project with abandonment option for $T=5$ years. We denote this value $M^{5}(V)$. For very high values of the outcome the value of the investment project with the abandonment option and the one with the switching option converge since for very high values of the outcome the option to abandon and to switch are far out of the money and therefore their values become negligible compared to the intrinsic values of the investment projects which are identical for the two models.

In Figure 3 we finally show the corresponding switching points on the horizontal axis for two of the three different values of the expected time to completion, denoted $S_{N}^{1}$ and $S_{N}^{5}$. The switching point for $T=10$ is to the right of the figure. The exact levels are $S_{N}^{1}=32.04, S_{N}^{5}=37.58$, and $S_{N}^{10}=44.79$.

In Figure 4 we show the switching points as a function of the expected time to completion, $T$, with our base case parameters. We denote the switching points $S_{N}$. Typically the switching points will be an increasing function of the expected time to completion but, as in the abandonment model, for short expected time to completion (for $T$ below 0.72 years with our base case parameters), it may be a decreasing function of time even for realistic parameter values. For $T=0$, the switching point is equal to $\frac{x_{1}}{x_{1}-1} K=35.75$ with our base case parameters as derived in Equation (22).

2.3. Model with both Abandonment and Switching Options. Next we consider the model where the owner of the investment project can switch between two activity levels, low and high. At the low activity level the on-going investment costs rate is $k$ and the intensity of completion is $\lambda$, and, hence, the expected time to completion is $T=\frac{1}{\lambda}$. At the high activity level the intensity of completion is increased to $\bar{\lambda}>\lambda$ but the on-going investment costs rate is also increased to $\bar{k}>k$. Note that at the high activity level the

\footnotetext{
${ }^{10}$ The size for the on-going investment costs rate, $k$, is irrelevant since the waiting time until completion is zero, so the present value of those costs will be zero. Note also that as $\lambda$ goes to infinity, $y_{2}$ will go to minus infinity.
} 




Figure 3. Values of investment projects (denoted $N^{1}(V), N^{5}(V)$, and $N^{10}(V)$ ) as a function of the value of the outcome, $V$, for three different values of expected time to completion, $T=1, T=5$, and $T=10$ years. The figure also shows the corresponding switching points (denoted $S_{N}^{1}$ and $S_{N}^{5}$ ) on the horizontal axis for two of the three different values of expected time to completion. The switching point for $T=10$ is off the chart. The exact levels are $S_{N}^{1}=32.04, S_{N}^{5}=37.58$, and $S_{N}^{10}=44.79$. For comparison the figure also shows the value of the investment project with the abandonment option (denoted $M^{5}(V)$ ) instead of the switching option for $T=5$ and its corresponding abandonment threshold level (denoted $\left.A_{M}^{5}\right)$. All other parameters are given in Table 1.

expected time to completion is reduced to $\bar{T}=\frac{1}{\lambda}<T$. When the value of the outcome is high, the owner will prefer to reduce the expected time to completion even if this requires a higher on-going investment costs rate, and when the value of the outcome is low, the owner will prefer to reduce the on-going investment costs rate even though the expected time to completion will be longer. Moreover, when the value of the outcome is very low the owner may even want to abandon the investment project. For the case of an R\&D investment project we can imagine that the owner can switch between two different levels of research activity. A low research activity level with low on-going costs rate, $k$, and a corresponding long expected time to completion, $T$, versus a high research activity level with higher on-going costs rate, $\bar{k}$, but also shorter expected time to completion, $\bar{T}$. The owner will of course also have the option to abandon the R\&D investment project definitively. Clearly the decision of which research activity level to choose and eventually when to abandon will depend on the net present value of the benefits from selling the products, $V$. As in the first model we 


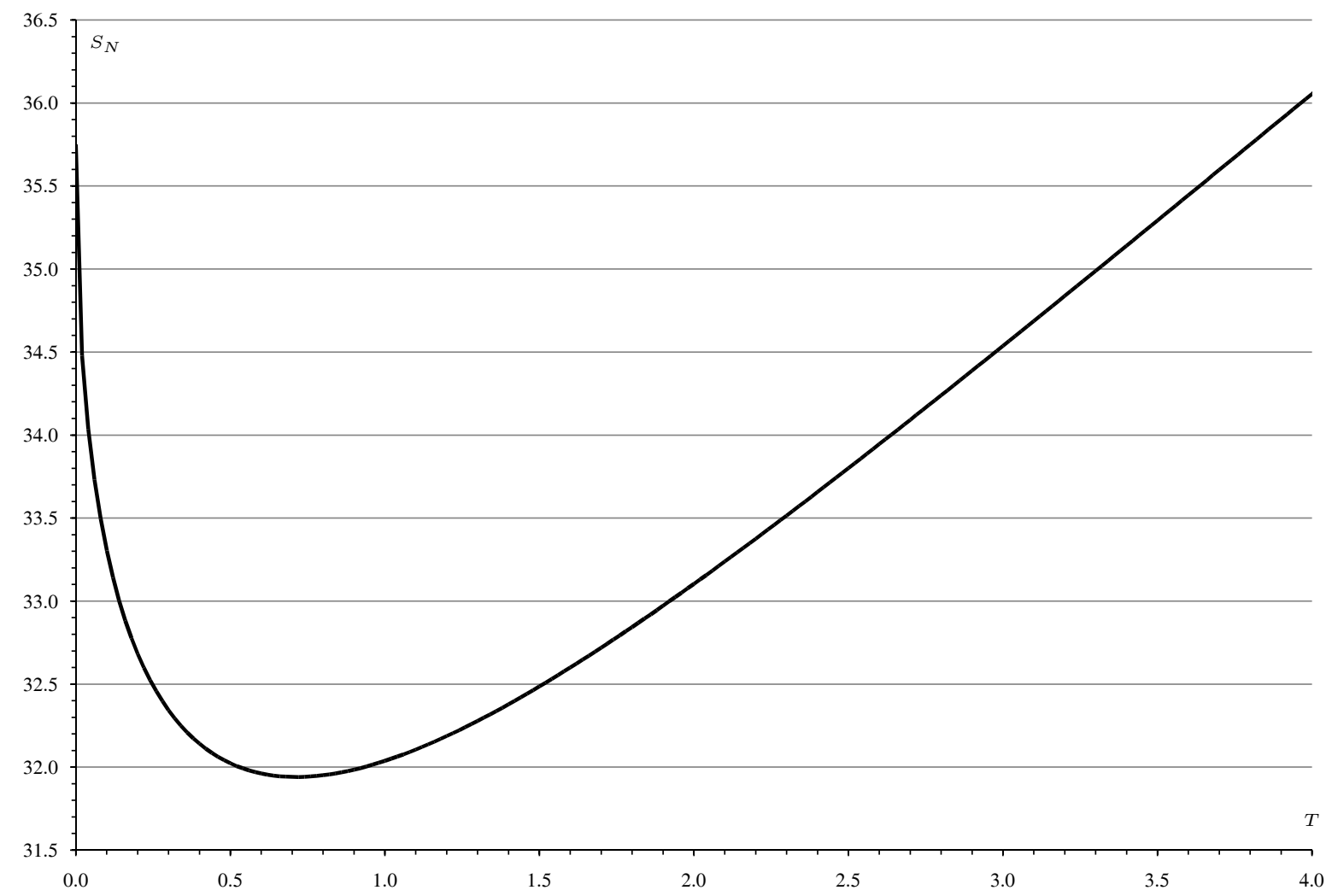

FIGURE 4. Switching points (denoted $S_{N}$ ) as a function of expected time to completion, $T$. All other parameters are given in Table 1.

studied in sub-section 2.1, there will be a threshold level, $A_{O}$, such that when the value of the outcome is below this level it is optimal for the owner of the investment project to abandon it; and as in the second model we studied in sub-section 2.2, there will be another threshold level, $S_{O}$, where it will be optimal to switch between the two possible activity levels. As in sub-section 2.2 we assume that there are no switching costs. We solve this model in Appendix B.

In Figure 5 we show the values of an investment project of this kind as a function of the value of the outcome, $V$. In this investment project the owner can switch to a lower level of activity with a lower ongoing investment costs rate, $k=0.3$, but also a longer expected time to completion, $T=10$ years. All other parameters are as in our base case. We denote this value $O(V)$. For this model the optimal switching point between the two activity levels is $S_{O}=51.41$ (which is to the right of the figure) and the optimal abandonment threshold level is $A_{O}=3.03$ with our base case parameters. For comparison we also plot the values of the corresponding investment project with only the abandonment option in the base case. That is, here the owner has expected time to completion of $T=5$ years and an on-going investment costs rate of $k=1$. In this model the owner's only option is to abandon her/his investment project, which she/he will do at the abandonment threshold level $A_{M}=5.26$. We denote this value $M(V)$. Also for comparison, we plot the values of the corresponding investment project with only the switching option in the base case. That is, here the owner can switch between a passive and an active investment project. The passive investment 


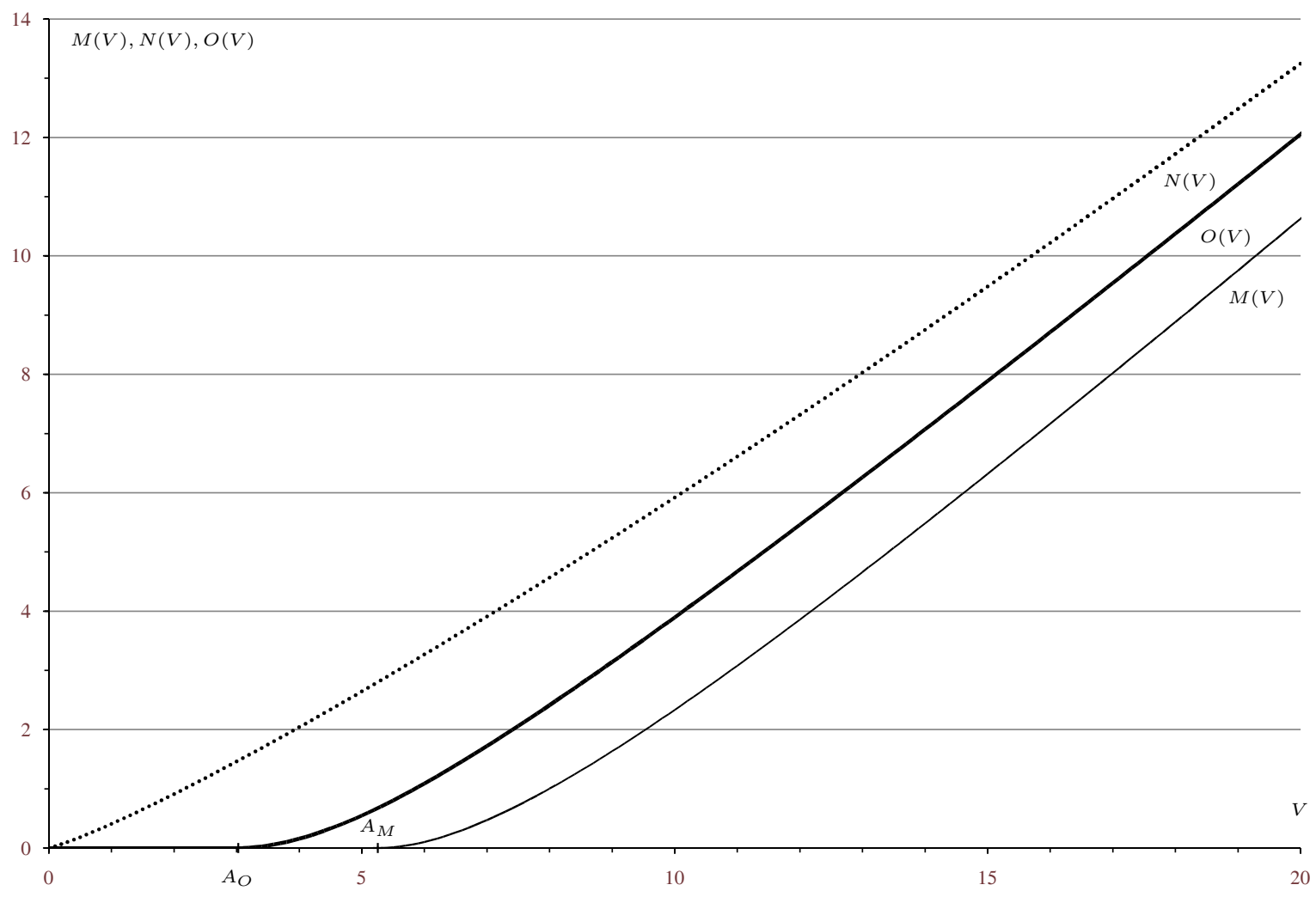

Figure 5. Values of an investment project (denoted $O(V)$ ) as a function of the value of the outcome, $V$, in which the owner can switch to a lower level of activity with a lower on-going investment costs rate, $k=0.3$, but also a longer expected time to completion, $T=10$ years. The optimal switching point between the two activity levels is $S_{O}=51.41$ and the optimal abandonment threshold level is $A_{O}=3.03$. For comparison the figure also shows the values of the corresponding investment project with only the abandonment option (denoted $M(V)$ ). The abandonment threshold level for this model is $A_{M}=5.26$. The figure also shows the values of the corresponding investment project with only the switching option (denoted $N(V)$ ). The switching point for this model is $S_{N}=37.58$. Both switching points $\left(S_{O}\right.$ and $\left.S_{N}\right)$ are off the chart. All other parameters are given in Table 1.

project has an on-going investment costs rate of $k=0$ and no chance of completion, i.e. $T=\infty$, whereas the active investment project has expected time to completion of $T=5$ years and an on-going investment costs rate of $k=1$. In this model the owner's only option is to switch between the passive and the active investment project, which she/he will do at the switching point $S_{N}=37.58$ (which is also to the right of the figure). We denote this value $N(V)$. Interestingly, as the value of the outcome, $V$, increases, the values of the three investment projects converge. This is so because for very high values of the outcome the option to abandon and/or switch are far out of the money and therefore their values become negligible compared to the intrinsic values of the investment projects which are identical for all three investment projects. 


\section{Duopoly Models}

In the previous section we considered models in which the owner of the investment project does not face any competition as to whom will have the right to the value of the outcome when the investment project is completed. Now we consider models in which there are two different owners of investment projects. Only the one whose project is completed first, gets the value of the outcome, $V$, and the other gets nothing. That is, we make a winner-takes-it-all assumption. A typical illustration of this would be when two competing research teams are working to develop similar products and the one who completes first is able to patent the product and obtain all the benefits. To simplify the analysis we consider models in which both owners (which we will now call players) have the same rate of on-going investment costs, $k$, the same final investment costs, $K$, and the same expected time to completion, $T$. We also assume that the two exponential distributions governing the time to completion of the two investment projects are independent. As in the previous section we will consider first the abandonment option and then the switching option.

3.1. Model with Abandonment Option. Since there is only one stochastic process governing the value of the outcome for both players and the two players have the same characteristics, both will meet the optimal abandonment threshold level at the same point in time. This abandonment threshold level will be as least as high as the abandonment threshold level in the corresponding monopoly model, $A_{M}$, from Equation (8b), which we derived in sub-section 2.1. Hence, both players will prefer that the other player abandons so that she/he would get the investment project as a monopolist. The decision as to whom abandons is the result of a game. To break the tie we first consider the case where one of the players, the leader, has an unmodeled advantage over the other player, the follower. This advantage could be slightly lower investment costs or more capital, etc. In this case, we assume that the follower abandons first and leaves the monopoly value to the leader. The threshold level at which the follower will abandon we will denote, $A_{Q}$. Clearly, as we have argued, $A_{Q} \geq A_{M}$. After having analyzed the leader-follower case we will consider the case where the two players are identical and analyze the game that occurs.

3.1.1. Leader and Follower. If the two players know in advance whom will be the player who will abandon first, the abandonment threshold levels will reflect this and be different for each of the two players.

3.1.1.1. The Follower. First we analyze the follower's investment project. As in the two previous abandonment models we analyzed in sub-sections 2.1 and 2.3 we will have to consider both the situation where we assume that the abandonment threshold level, $A_{Q}$, is below the final investment costs, $K$, and the situation where it is above. Here we assume that $A_{Q}<K$. In Appendix $\mathrm{C}$ we analyze the situation when $A_{Q} \geq K$. Let $Q(V)$ denote the value of the investment project for the follower. This value must satisfy the system of ODEs

$$
\frac{1}{2} \sigma^{2} V^{2} Q^{\prime \prime}(V)+\mu V Q^{\prime}(V)-(r+2 \lambda) Q(V)-k=0 \quad \text { when } A_{Q}<V<K,
$$

and

$$
\frac{1}{2} \sigma^{2} V^{2} Q^{\prime \prime}(V)+\mu V Q^{\prime}(V)-(r+2 \lambda) Q(V)-k+\lambda(V-K)=0 \quad \text { when } K<V .
$$

Equation (24a) describes the value of the follower's investment project when it is out of the money. This equation reflects the fact that with intensity $2 \lambda$ either the follower's or the leader's investment project will be completed out of the money. If it is the follower's investment project which is completed out of the money the 
value of her/his investment project would jump down to zero (cf. footnote 6). If it is the leader's investment project which is completed out of the money, we assume that the value of the follower's investment project also jumps down to zero. ${ }^{11}$ In addition, the follower has to pay on-going investment costs at the rate $k$ per unit of time to keep the investment project alive. Equation (24b) describes the value of follower's investment project when it is in the money. This equation reflects the fact that with intensity $2 \lambda$ either the follower's or the leader's investment project will be completed in the money. If it is the follower's investment project which is completed, the value of her/his investment project would jump to the completion value, $V-K$. But, if it the leader's investment project which is completed, the value of the follower's investment project will jump to zero because of our winner-takes-it-all assumption. In addition, the follower has to pay on-going investment costs at the rate $k$ per unit of time to keep the investment project alive. The general solutions to each of the two ODEs, (24a) and (24b), which we denote $Q_{1}(V)$ and $Q_{2}(V)$, are

$$
Q_{1}(V)=q_{11} V^{z_{1}}+q_{12} V^{z_{2}}-\frac{k}{r+2 \lambda} \quad \text { when } A_{Q} \leq V \leq K
$$

and

$$
Q_{2}(V)=q_{21} V^{z_{1}}+q_{22} V^{z_{2}}+\frac{\lambda V}{r+2 \lambda-\mu}-\frac{k+\lambda K}{r+2 \lambda} \quad \text { when } K \leq V .
$$

Here the powers, $z_{1}$ and $z_{2}$, are given by

$$
z_{1}=\frac{\left(\frac{1}{2} \sigma^{2}-\mu\right)+\sqrt{\left(\mu-\frac{1}{2} \sigma^{2}\right)^{2}+2(r+2 \lambda) \sigma^{2}}}{\sigma^{2}}>1
$$

and

$$
z_{2}=\frac{\left(\frac{1}{2} \sigma^{2}-\mu\right)-\sqrt{\left(\mu-\frac{1}{2} \sigma^{2}\right)^{2}+2(r+2 \lambda) \sigma^{2}}}{\sigma^{2}}<0 .
$$

Comparing the powers $z_{1}$ and $z_{2}$ with the corresponding powers $y_{1}$ and $y_{2}$ from the corresponding monopoly model from the system of equations (5), we see that $z_{1}>y_{1}>1$ and that $z_{2}<y_{2}<0$. Using the same arguments as for the previous models, we know that $\lim _{V \rightarrow \infty} \frac{Q_{2}(V)}{V}$ must be finite. Hence, we can eliminate the $V^{z_{1}}$ term in Equation (25b). In addition, we can substitute $T=\frac{1}{\lambda}$ for the expected (remaining) time to completion of the investment project to get

$$
Q_{1}(V)=q_{11} V^{z_{1}}+q_{12} V^{z_{2}}-\frac{k T}{2+r T} \quad \text { when } A_{Q} \leq V \leq K
$$

and

$$
Q_{2}(V)=q_{22} V^{z_{2}}+\frac{V}{2+(r-\mu) T}-\frac{k T+K}{2+r T} \quad \text { when } K \leq V .
$$

\footnotetext{
${ }^{11}$ This reflects that the leader still has a right to her/his discovery (either by patenting or other legal actions) even though it turns out that it is not worth putting into production immediately. As we indicated in footnote 6 on page 6 a more realistic assumption would be that the owner (in this case the leader) receives a perpetual American option at the completion date of her/his investment project. The important assumption here is that if the leader's investment project is completed, then the follower's investment project becomes valueless because of the winner-takes-it-all assumption. We think this is the most realistic and appealing assumption. As an alternative, we could assume that if the leader's investment project is completed out of the money, then the follower suddenly becomes a monopolist and that the value of her/his investment project will jump to the corresponding monopoly value, $M(V)$, which we derived in sub-section 2.1. This case is also solvable, although the optimal abandonment threshold level can only be found numerically.
} 
The value of the follower's investment project has the following boundary conditions

$$
\begin{aligned}
Q_{1}\left(A_{Q}\right) & =0, \\
Q_{1}^{\prime}\left(A_{Q}\right) & =0, \\
Q_{1}(K) & =Q_{2}(K),
\end{aligned}
$$

and

$$
Q_{1}^{\prime}(K)=Q_{2}^{\prime}(K)
$$

These boundary conditions are the same as the boundary conditions used in the corresponding monopoly model with abandonment, cf. the system of equations (7) in sub-section 2.1. In order to find solutions for the four unknowns, $q_{11}, q_{12}, q_{22}$, and $A_{Q}$, we solve the system of equations (28). The solution is

$$
\begin{aligned}
q_{11} & =\frac{\left(2+\left(r-z_{2} \mu\right) T\right) K^{1-z_{1}}}{\left(z_{1}-z_{2}\right)(2+(r-\mu) T)(2+r T)}, \\
A_{Q} & =\left(\frac{z_{2} k T}{\left(z_{2}-z_{1}\right)(2+r T) q_{11}}\right)^{\frac{1}{z_{1}}}, \\
q_{12} & =-\frac{z_{1} q_{11} A_{Q}^{z_{1}-z_{2}}}{z_{2}},
\end{aligned}
$$

and

$$
q_{22}=q_{12}+\frac{\left(2+\left(r-z_{1} \mu\right) T\right) K^{1-z_{2}}}{\left(z_{1}-z_{2}\right)(2+(r-\mu) T)(2+r T)} .
$$

Finally, the value of the follower's investment project is

$$
Q(V)= \begin{cases}0 & \text { when } V<A_{Q}, \\ q_{11} V^{z_{1}}+q_{12} V^{z_{2}}-\frac{k T}{2+r T} & \text { when } A_{Q} \leq V<K, \\ q_{22} V^{z_{2}}+\frac{V}{2+(r-\mu) T}-\frac{k T+K}{2+r T} & \text { when } K \leq V .\end{cases}
$$

Note the close similarity to the corresponding monopoly solution from Equation (9) and the system of equations (8) in sub-section 2.1. It is not so obvious when one looks at the ODE, but from the solution it is clear that the value of the follower's investment project is identical to half the value of the corresponding monopoly investment project with double the on-going investment costs rate and half the expected time to completion. ${ }^{12}$ Therefore, the abandonment threshold level for the follower, $A_{Q}$, is also identical to the

\footnotetext{
${ }^{12}$ Ex post, after having discovered this result, it is easy to see that it is possible to derive it directly from the ODEs. Take the system of ODEs (24) describing the value of the follower's investment project, $Q(V)$. In order to get two times this solution, i.e. $2 Q(V)$, we need to double the cash flow rates. That is, $X(V)=2 Q(V)$ would be a solution to the system of ODEs

$$
\frac{1}{2} \sigma^{2} V^{2} X^{\prime \prime}(V)+\mu V X^{\prime}(V)-(r+2 \lambda) X(V)-2 k=0 \quad \quad \text { when } A_{X}<V<K,
$$

and

$$
\frac{1}{2} \sigma^{2} V^{2} X^{\prime \prime}(V)+\mu V X^{\prime}(V)-(r+2 \lambda) X(V)-2 k+2 \lambda(V-K)=0 \quad \text { when } K<V .
$$

The boundary conditions for $X(V)$ are the same as for $Q(V)$, cf. Equations (28). Now comparing the system of ODEs (31) to the corresponding system of ODEs (3) for the monopoly model from sub-section 2.1 and also the boundary conditions, cf. Equations (7), it is clear that $X(V)$ is the value of a monopoly investment project with intensity $2 \lambda$ (corresponding to an expected time to completion of $\frac{T}{2}$ ) and an on-going investment costs rate of $2 k$.
} 
abandonment threshold level for the corresponding monopoly investment project with double the on-going investment costs rate and half the expected time to completion.

If it turns out that the solution to $A_{Q}$ from Equation (29b) is greater than $K$, we must reconsider the problem. We do this in Appendix C. In this situation, the value of the follower's investment project is

$$
Q(V)= \begin{cases}0 & \text { when } V<\tilde{A}_{Q}, \\ q_{32} V^{z_{2}}+\frac{V}{2+(r-\mu) T}-\frac{k T+K}{2+r T} & \text { when } \tilde{A}_{Q} \leq V .\end{cases}
$$

Here $\tilde{A}_{Q}$ and $q_{32}$ are

$$
\tilde{A}_{Q}=\frac{z_{2}(2+(r-\mu) T)(k T+K)}{\left(z_{2}-1\right)(2+r T)}
$$

and

$$
q_{32}=-\frac{\tilde{A}_{Q}^{1-z_{2}}}{z_{2}(2+(r-\mu) T)} .
$$

Note again that the abandonment threshold level in this situation (denoted $\tilde{A}_{Q}$ ) is, in general, different from $A_{Q}$ from Equation (29b). For notational simplicity, however, we will always refer to $A_{Q}$ as the abandonment threshold level for the follower for the rest of the paper even though strictly speaking we should distinguish between $A_{Q}$ and $\tilde{A}_{Q}$ depending on whether $A_{Q}<K$ or $\tilde{A}_{Q} \geq K$.

3.1.1.2. The Leader. After having found the abandonment threshold level for the follower, $A_{Q}$, we can turn to the valuation of the leader's investment project. The abandonment threshold level for the leader, $A_{P}$, will always be lower than the abandonment threshold level for the follower, $A_{Q}$. Hence, the follower will always have abandoned before the leader considers abandoning her-/himself. So the leader will find her-/himself in the corresponding monopoly situation before she/he considers abandoning. Hence, the abandonment threshold level for the leader will be the same as the abandonment threshold level in the corresponding monopoly model. That is, $A_{P}=A_{M}$. Moreover, the value of the leader's investment project will be equal to the value of the corresponding investment project in the monopoly model, $M(V)$, as soon as the follower has abandoned. Hence, we only need to analyze the value of the leader's investment project for values of the outcome, $V$, above the abandonment threshold level for the follower, $A_{Q}$. As it was the case when we analyzed the value of the follower's investment project, we have to consider both the situation when $A_{Q}<K$ and and when $A_{Q} \geq K$. Here we analyze the situation when $A_{Q}<K$. In Appendix D we analyze the situation when $A_{Q} \geq K$. Let $P(V)$ denote the value of the leader's investment project. This value must satisfy the system of ODEs

$$
\frac{1}{2} \sigma^{2} V^{2} P^{\prime \prime}(V)+\mu V P^{\prime}(V)-(r+2 \lambda) P(V)-k=0 \quad \text { when } A_{Q}<V<K,
$$

and

$$
\frac{1}{2} \sigma^{2} V^{2} P^{\prime \prime}(V)+\mu V P^{\prime}(V)-(r+2 \lambda) P(V)-k+\lambda(V-K)=0 \quad \text { when } K<V .
$$

The system of equations (34) for the value of the leader's investment project is identical to the system of equations (24) for the value of the follower's investment project and the explanations for the terms are the same. Since the value of the leader's investment project is equal to the value of the corresponding investment 
project in the monopoly model as soon as the follower has abandoned we have

$$
P(V)=M(V) \quad \text { when } A_{M} \leq V \leq A_{Q} .
$$

Using the same arguments as for the follower we get the solutions to each of the two ODEs, (34a) and (34b), which we denote $P_{1}(V)$ and $P_{2}(V)$, as

$$
P_{1}(V)=p_{11} V^{z_{1}}+p_{12} V^{z_{2}}-\frac{k T}{2+r T} \quad \text { when } A_{Q} \leq V \leq K
$$

and

$$
P_{2}(V)=p_{22} V^{z_{2}}+\frac{V}{2+(r-\mu) T}-\frac{k T+K}{2+r T} \quad \text { when } K \leq V .
$$

The value of the leader's investment project has the following boundary conditions

$$
\begin{gathered}
P_{1}\left(A_{Q}\right)=M\left(A_{Q}\right), \\
P_{1}(K)=P_{2}(K),
\end{gathered}
$$

and

$$
P_{1}^{\prime}(K)=P_{2}^{\prime}(K)
$$

Condition (36a) reflects the fact that at the abandonment threshold level for the follower, $A_{Q}$, the value of the leader's investment project is the same as the value of the corresponding monopoly investment project. Note that the value of the leader's investment project is not (necessarily) differentiable at the abandonment threshold level for the follower. This is so because (i) the leader does not take any active decisions at this point, ${ }^{13}$ and (ii) the value process of the leader's investment project only follows this path once (opposite a switching point where the value processes of the two players' investment projects may pass back and forth over this threshold level infinitely many times). Conditions (36b) and (36c) are the usual continuity and differentiability conditions at the final investment costs, $K$. We can solve the system of equations (36) in the three unknowns, $p_{11}, p_{12}$, and $p_{22}$. The solution is ${ }^{14}$

$$
\begin{aligned}
& p_{11}=\frac{\left(2+\left(r-z_{2} \mu\right) T\right) K^{1-z_{1}}}{\left(z_{1}-z_{2}\right)(2+(r-\mu) T)(2+r T)}, \\
& p_{12}=\left(m_{11} A_{Q}^{y_{1}}+m_{12} A_{Q}^{y_{2}}-p_{11} A_{Q}^{z_{1}}-\frac{k T}{(1+r T)(2+r T)}\right) \frac{1}{A_{Q}^{z_{2}}},
\end{aligned}
$$

and

$$
p_{22}=p_{12}+\frac{\left(2+\left(r-z_{1} \mu\right) T\right) K^{1-z_{2}}}{\left(z_{1}-z_{2}\right)(2+(r-\mu) T)(2+r T)} .
$$

Note that the solution to $p_{11}$ from Equation (37a) is identical to $q_{11}$ for the follower from Equation (29a). Note also the similarity between $p_{22}$ from Equation (37c) and $q_{22}$ for the follower from Equation (29d).

\footnotetext{
${ }^{13}$ It is the follower who determines when to abandon her/his investment project, so her/his value should smooth paste at this threshold level, cf. Equation (28b).

${ }^{14}$ Note that $M(V)$ on the right hand side of Equation (36a) is actually $M_{1}(V)$ from Equation (6a), since $A_{M}<A_{Q}<K$. Therefore we get the terms $m_{11}$ and $m_{12}$ in the solution to $p_{12}$ in Equation (37b).
} 
Finally, the value of the leader's investment project is

$$
P(V)= \begin{cases}0 & \text { when } V<A_{M}, \\ M(V) & \text { when } A_{M} \leq V<A_{Q}, \\ p_{11} V^{z_{1}}+p_{12} V^{z_{2}}-\frac{k T}{2+r T} & \text { when } A_{Q} \leq V<K, \\ p_{22} V^{z_{2}}+\frac{V}{2+(r-\mu) T}-\frac{k T+K}{2+r T} & \text { when } K \leq V .\end{cases}
$$

We analyze the situation when $A_{Q}$ from Equation (29b) is greater than $K$ in Appendix D. In this situation the value of the leader's investment project is

$$
P(V)= \begin{cases}0 & \text { when } V<A_{M}, \\ M(V) & \text { when } A_{M} \leq V<\tilde{A}_{Q}, \\ p_{32} V^{z_{2}}+\frac{V}{2+(r-\mu) T}-\frac{k T+K}{2+r T} & \text { when } \tilde{A}_{Q} \leq V .\end{cases}
$$

Here $p_{32}$ is

$$
p_{32}= \begin{cases}\left(m_{22} \tilde{A}_{Q}^{y_{2}}-\frac{k T+K}{(1+r T)(2+r T)}+\frac{\tilde{A}_{Q}}{(1+(r-\mu) T)(2+(r-\mu) T)}\right) \frac{1}{\tilde{A}_{Q}^{z_{2}}} & \text { when } A_{M}<K \\ \left(m_{32} \tilde{A}_{Q}^{y_{2}}-\frac{k T+K}{(1+r T)(2+r T)}+\frac{\tilde{A}_{Q}}{(1+(r-\mu) T)(2+(r-\mu) T)}\right) \frac{1}{\hat{A}_{Q}^{22}} & \text { when } A_{M} \geq K .\end{cases}
$$

Substituting the value of the corresponding monopoly investment project, $M(V)$, into Equation (39) is straight forward but a little care is needed. ${ }^{15}$ See Appendix D for details.

In figure 6 we show the values of both the leader's and the follower's investment projects as a function of the value of the outcome, $V$, with our base case parameters. We denote the values of the leader's investment project as $P(V)$ and the values of the follower's investment project as $Q(V)$. For comparison we also show the values of the corresponding monopoly investment project. These values we denote $M(V)$. We also show the corresponding abandonment threshold levels on the horizontal axis for the follower and for the leader. We denote the abandonment threshold level for the follower $A_{Q}$. The abandonment threshold level for the leader is the same as the abandonment threshold level in the corresponding monopoly model. Therefore, we denote this abandonment threshold level, $A_{M}$. The exact levels are $A_{Q}=6.30$ and $A_{M}=5.26$ with our base case parameters. For values of the outcome below the abandonment threshold level for the follower, $A_{Q}$, the value of the leader's investment project is the same as the value of the corresponding monopoly investment project.

It is interesting to note that the value of the leader's investment project is actually a decreasing function of the value of the outcome for values of the outcome just above the abandonment threshold level for the follower (for $V$ between 6.30 and 6.56 with our base case parameters). The reason for this is as follows: Suppose we are in the situation when the follower has not yet abandoned. A small increase in the value of the outcome, $V$ has two effects. There is a value decreasing effect on the leader's investment project because of the reduced likelihood that the follower will abandon and there is a (natural) value increasing effect of a higher value of the outcome at completion of the leader's investment project. For values of the outcome close to the threshold level where the follower will abandon, the value decreasing effect dominates.

\footnotetext{
${ }^{15}$ Basically, one needs to be careful to substitute the correct version of $M(V)$ into Equation (39) depending on whether $A_{M}<K$ or $A_{M} \geq K$.
} 


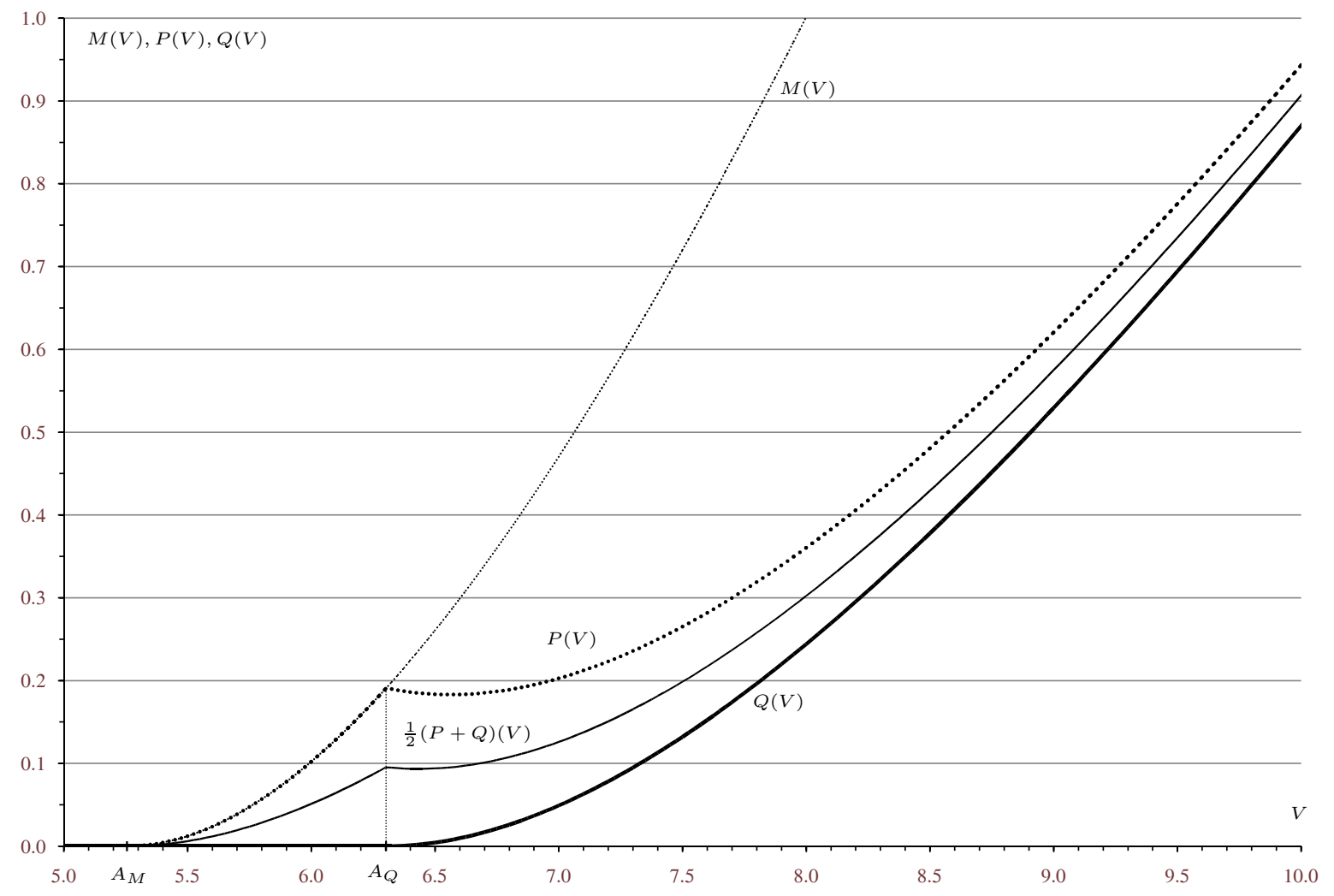

Figure 6. Values of both the leader's (denoted $P(V)$ ) and the follower's (denoted $Q(V)$ ) investment projects as a function of the value of the outcome, $V$. For comparison the figure also shows the values of the corresponding monopoly investment project (denoted $M(V)$ ). The figure also shows the values of the investment projects using the coin-toss solution (denoted $\frac{1}{2}(P+Q)(V)$ ), cf. Paragraph 3.1.2.1 below. Finally, the figure also shows the corresponding abandonment threshold levels on the horizontal axis for the follower (denoted $\left.A_{Q}\right)$ and for the leader (denoted $A_{M}$ ). The exact levels are $A_{Q}=6.30$ and $A_{M}=5.26$. All other parameters are given in Table 1 .

For high levels of the outcome the values for the leader's and the follower's investment projects converge because the advantage of being the leader becomes relatively small compared to the intrinsic values of the investment projects which are identical for the leader's and the follower's investment projects.

3.1.2. Identical Players. When the two players are truly identical we need to analyze the abandonment decision as a game. The value of each of the two players' investment projects when both keep investing we will denote $\hat{R}(V) . M(V)$ is the value of the investment project in the corresponding monopoly model from sub-section 2.1. It is easy to see that $\hat{R}(V) \leq M(V)$. The two players consider whether to continue investing or whether to abandon by studying the normal form representation of the game in Table 2. If the value of the outcome, $V$, is high enough to make $\hat{R}(V)$ strictly positive, it is easy to see (by eliminating strictly dominated strategies) that the unique Nash equilibrium in pure strategies is that both players continue investing in their investment projects. When the value of the outcome is below the abandonment threshold level in the corresponding monopoly model, i.e. when $V \leq A_{M}$, it is no longer profitable to continue investing 


\begin{tabular}{|c|c|c|c|}
\hline & \multicolumn{2}{|c|}{ Player two } \\
\hline & & Continues investing & Abandons \\
\hline \multirow{2}{*}{ Player one } & \multirow{2}{*}{$\begin{array}{c}\text { Continues investing } \\
\text { Abandons }\end{array}$} & $\hat{R}\left(V_{t}\right), \hat{R}\left(V_{t}\right)$ & $M\left(V_{t}\right), 0$ \\
\hline & & $0, M\left(V_{t}\right)$ & 0,0 \\
\hline
\end{tabular}

TABLE 2. Normal form representation of the (one-shot) abandonment decision game. $\hat{R}(V)$ denotes the value of each of the two players' investment projects when both keep investing. $M(V)$ denotes the value of the investment project in the corresponding monopoly model from sub-section 2.1.

even for a monopolist, so then the unique Nash equilibrium in pure strategies is that both players abandon their investment projects. Finally, when the value of the outcome is in the area when $\hat{R}(V)<0<M(V)$, then there are two Nash equilibria in pure strategies. These are characterized by the fact that one of the players continues investing and the other player abandons. This is exactly the leader-follower case, which we have just analyzed. However, if the players are (truly) identical there is no mechanism to select one of the two equilibria from the other. A co-operative solution in this case would be that the two players agree to meet and to flip a coin in order to break the tie and decide whom should be the leader and whom should be the follower. We will term this solution the Coin Toss Solution. From an ex-ante point of view both players prefer this solution, since this gives each of the two players the highest ex-ante value of their individual investment projects.

3.1.2.1. Coin-Toss Solution. If the players have agreed that they will meet to flip a coin in order to determine whom should be the leader and whom should be the follower, and it is clear that the looser ex post, after the coin has been tossed, would honor the agreement and actually abandon, then both players would like to know the outcome of the coin toss as soon as the value of the outcome, $V$, hits (or is below) the abandonment threshold level for the follower, $A_{Q}$, from Equation $(29 \mathrm{~b}),{ }^{16}$ in the corresponding leader-follower case. This is so because $A_{Q}$ is the optimal abandonment threshold level for the looser of the coin toss. As long as $V$ is above $A_{Q}$ the players do not need to know the outcome of the coin toss since it would not change their investment decisions - both players would optimally continue investing for values of the outcome, $V$, above $A_{Q}$.

Before the players flip the coin to determine whom should be the follower and whom should be the leader they each have a fifty-fifty chance of being the leader or being the follower. Hence, the value of each of the two players' investment projects in this case will be half the value of the leader's investment project and half the value of the follower's investment project from the corresponding leader-follower case.

In Figure 6 we show the values of the investment projects using the coin-toss solution as a function of the value of the outcome, $V$, with our base case parameters. We denote these values $\frac{1}{2}(P+Q)(V)$. Obviously, these are the values of each of the two players' investment projects for a given value of the outcome before the coin is flipped. At the point in time when the coin is flipped the value of the winner's investment project jumps up to the value of the leader's investment project, $P(V)$, and the value of the looser's investment

${ }^{16}$ Note that if $A_{Q}$ from Equation (29b) is above the fixed investment costs, $K$, then the relevant abandonment threshold level for the follower is $\tilde{A}_{Q}$ from Equation (33a) and not $A_{Q}$ from Equation (29b). 
project jumps down to the value of the follower's investment project, $Q(V)$. It is interesting to note that also the values of the investment projects using the coin-toss solution is a decreasing function of the value of the outcome for values of the outcome just above the abandonment threshold level for the follower, $A_{Q}$, (for $V$ between 6.30 and 6.44 with our base case parameters). However, the interval in which the values of the investment projects using the coin-toss solution is a decreasing function of the value of the outcome is smaller than the interval where the values of the leader's investment project is a decreasing function of the values of the outcome because the values of the follower's investment project is always an increasing function of the value of the outcome.

For values of the outcome in the interval between the abandonment threshold levels for the leader and for the follower, i.e., for $V \in\left(A_{M}, A_{Q}\right)$, the interpretation of the values $\frac{1}{2}(P+Q)(V)$ is still that it is the values to each of the two players before the coin is flipped. One reason why these values can be of interest is that the initial date-zero value of $V$ may be in this range, i.e., $V_{0} \in\left(A_{M}, A_{Q}\right)$. In this case the players should flip the coin immediately and the looser should abandon as soon as the outcome is realized.

3.1.2.2. Variable Abandonment Intensity Solution. A problem with the Coin-Toss Solution, however, is that it is not sequentially rational for the looser of the coin toss to actually abandon if she/he thinks that the whole game can be repeated if she/he stays in. In continuous time repeated games can be defined in different ways. The basic problem is that if there is not at least some finite time interval between points in time when the game outlined in Table 2 can be repeated, then there is basically no costs of playing another round and therefore none of the players will ever abandon. That is, we are not able to trade off the present value of the on-going costs of keeping investing (for another round) and the present value (of the probability) of becoming the monopolist.

One way to introduce this trade off is to let each player choose an abandonment intensity at each instant in time. It turns out that intensities play the same role in a continuous-time model as mixed strategies does in a non-repeated game or even in a repeated game setting in discrete time. A player's choice of an abandonment intensity (i.e., a probability to abandon per unit of time) quantifies her/his tendency to abandon versus continuing investing in her/his investment project in the right way in a continuous-time model since all the benefits and costs are also in rates per unit of time.

To be precise, we assume that each player has an associated Cox process with an intensity chosen by the player. This intensity may vary over time. However, since the only state variable in our model is the value of the outcome, $V$, and this process is Markovian, the intensity can at most depend on the current realization of this process. We denote player $i$ 's chosen abandonment intensity $\gamma_{i}(V)$, for $i=1,2$. The strategy of player $i$ is to continue investing in her/his investment project until the associated Cox process jumps. When the Cox process jumps, player $i$ should abandon her/his investment project. That is, player $i$ abandons her/his investment project with (instantaneous) probability $\gamma_{i}(V)$ per unit of time. We assume that, conditional on $V$, the two Cox processes governing the actual abandonments of each of the two players are independent. Player $i$ 's strategy is completely determined by her/his chosen abandonment intensity, $\gamma_{i}(V)$. Hence, we will call $\gamma_{i}(V)$ player $i$ 's abandonment strategy.

For values of the outcome, $V$, above the abandonment threshold level for the follower, $A_{Q}$, in the corresponding leader-follower case both players will continue investing for sure so they will both optimally choose to have zero abandonment intensities, i.e., $\gamma_{i}(V)=0$ for $V>A_{Q}$ and $i=1$, 2. For values of the outcome, $V$, at or below the abandonment threshold level in the corresponding monopoly model, $A_{M}$, both players 
will abandon immediately so they will both optimally choose infinitely high abandonment intensities, i.e., $\gamma_{i}(V)=\infty$ for $V \leq A_{M}$ and $i=1,2$. For values of the outcome, $V$, in the interval between $A_{M}$ and $A_{Q}$, both players will optimally choose non-trivial abandonment intensities which trades off the on-going costs of keeping investing in their individual investment projects and their individual value of (the probability of) becoming the monopolist. Clearly, as soon as one of the players abandons her/his investment project, the other player automatically becomes the monopolist, and therefore, it is optimal for her/him to switch her/his abandonment intensity to zero.

The interesting question is how the two players should choose their individual abandonment strategies, $\gamma_{i}(V)$, for $i=1,2$, as their (instantaneous) responses in an instantaneously repeated game in continuous time for all possible values of the outcome, $V$, in the (relevant) interval between $A_{M}$ and $A_{Q}$.

Let $R_{i}(V)$ denote the value of player $i$ 's investment project in this case, $i=1,2$. Note that this value, in general, will depend on both players chosen abandonment strategies, $\gamma_{j}(V), j=1,2$.

An essential argument in our approach to finding the abandonment strategies is that the chosen strategies should be sequentially rational. That is, when the associated Cox process of one of the players, say player $i$, jumps, indicating that player $i$ should abandon her/his investment project, then it is essential that abandonment is not a dominated strategy for player $i$. If player $i$ 's value of continuing investing in her/his investment project, $R_{i}(V)$, is strictly positive, then abandonment (which clearly has value zero) would be dominated by continuing investing for player $i$. Hence, player $i$ 's value of continuing investing in her/his investment project cannot be strictly positive. On the other hand, if player $i$ 's value of continuing investing in her/his investment project is strictly negative, then the strategy of continued investing (with the chosen abandonment intensities for both players, $\left.\gamma_{i}(V), i=1,2\right)$ would be strictly dominated by immediate abandonment (corresponding to an infinitely high abandonment intensity) for player $i$. That is, the chosen abandonment intensity for the players, $\gamma_{i}(V), i=1,2$, for values of the outcome, $V$, in the interval between $A_{M}$ and $A_{Q}$ can only lead to sequentially rational strategies if $R_{i}(V)=0$ for all values of the outcome, $V$, in the interval between $A_{M}$ and $A_{Q}$ and $i=1,2$.

As it was the case in all the previous abandonment models we have analyzed in this paper, we have to consider different situations, depending on where the threshold levels for abandonment are compared to the final investment costs, $K$. For this case we will have three situations to analyze. We will have (i) $A_{M}<A_{Q}<K$, (ii) $A_{M}<K \leq \tilde{A}_{Q}$, and (iii) $K \leq \tilde{A}_{M}<\tilde{A}_{Q}$. Here we analyze the first situation when $A_{M}<A_{Q}<K$. In Appendix $\mathrm{F}$ we analyze the two other situations, which are conceptually similar.

As we have just argued, $R_{i}(V)=0$ for $V \in\left(A_{M}, A_{Q}\right]$ and $i=1,2$. On the other hand, using arguments similar to all the other models analyzed in this paper, since player $i$ is still investing in her/his investment project, $R_{i}(V)$ is still a solution to the ODE

$$
\begin{aligned}
\frac{1}{2} \sigma^{2} V^{2} R_{i}^{\prime \prime}(V)+\mu V R_{i}^{\prime}(V)-\left(r+2 \lambda+\gamma_{i}(V)+\right. & \left.\gamma_{j}(V)\right) R_{i}(V) \\
& +\gamma_{j}(V) M_{i}(V)-k_{i}=0 \quad \text { when } A_{M}<V<A_{Q} .
\end{aligned}
$$

Here we use index $j$ to refer to the other player, i.e. $j=3-i . M_{i}(V)$ denotes player $i$ 's value of the investment project in the corresponding monopoly model from sub-section 2.1. ${ }^{17}$ Since both players assign the same value to the investment project in the corresponding monopoly model there is, strictly speaking, no

\footnotetext{
${ }^{17}$ In sub-section 2.1 we used subscripts on $M(V)$ to denote the different general solutions to the different ODEs in the system of ODEs (3). Here we use subscripts to denote player specific values of becoming the monopolist.
} 
need for the index $i$ on the value $M(V)$, but we think it clarifies our arguments if we allow for player specific values of becoming the monopolist. For the same reason, we also allow for a player specific rate of on-going investment costs, $k_{i}$, in ODE (41). The ODE (41) describes the value of player $i$ 's investment project when it is out of the money. The ODE has the same terms as the ODE (24a) for the value of the follower's investment project and the ODE (34a) for the value of the leader's investment project in the corresponding leader-follower case and the explanations to these terms are the same. In addition, the ODE (41) has two extra terms, $\left(\gamma_{i}(V)+\gamma_{j}(V)\right) R_{i}(V)$ and $\gamma_{j}(V) M_{i}(V)$. The term $\left(\gamma_{i}(V)+\gamma_{j}(V)\right) R_{i}(V)$ reflects the fact that with intensity $\gamma_{i}(V)+\gamma_{j}(V)$ either player $i$ or player $j$ will abandon. If it is player $i$ who abandons then the value of her/his investment project clearly jumps to zero. This corresponds to a loss in value of $R_{i}(V)$. If it is player $j$ who abandons, then player $i$ becomes the monopolist and therefore the value of her/his investment project jumps to $M_{i}(V)$. This corresponds to a jump in value of $M_{i}(V)-R_{i}(V)$. This explains the second term, $\gamma_{j}(V) M_{i}(V)$.

Since $R_{i}(V)$ is zero over the whole interval $\left(A_{M}, A_{Q}\right]$, we know that both $R_{i}^{\prime}(V)$ and $R_{i}^{\prime \prime}(V)$ must also be zero in the whole open interval $\left(A_{M}, A_{Q}\right)$. Hence, from Equation (41) we conclude that the only way that the chosen strategies for the two players can be sequentially rational is if player $j$ chooses her/his abandonment strategy to be

$$
\gamma_{j}^{*}(V)= \begin{cases}\infty & \text { when } V \leq A_{M}, \\ \frac{k_{i}}{M_{i}(V)} & \text { when } A_{M}<V \leq A_{Q}, \\ 0 & \text { when } A_{Q}<V .\end{cases}
$$

Clearly the same arguments apply to player $i$ 's abandonment strategy. So player $i$ chooses her/his abandonment strategy to be $\gamma_{i}^{*}(V)$ from Equation (42).

The question is now whether the abandonment strategies $\left(\gamma_{1}^{*}(V), \gamma_{2}^{*}(V)\right)$, from Equation (42), form a Nash equilibrium. We show formally that this is the case in Appendix E. Basically the argument is that as long as both players use the abandonment strategies, $\gamma_{i}^{*}(V), i=1,2$, both players' values of their individual investment projects, $R_{i}(V)$, are zero. The question is now whether any of the players, say player $i$, has any incentives to deviate from using the abandonment intensity $\gamma_{i}^{*}(V)$, given that she/he knows that the other player (player $j$ ) uses the abandonment intensity $\gamma_{j}^{*}(V)$. When player $i$ 's value of her/his investment project, $R_{i}(V)$, is zero, and she/he knows that player $j$ uses the abandonment intensity, $\gamma_{j}^{*}(V)$, then player $i$ is instantaneously indifferent between any choice of her/his own abandonment intensity-including immediate abandonment. So there is no other strategy, i.e. no other choice of abandonment intensity, for player $i$ that is strictly better than using the abandonment intensity, $\gamma_{i}^{*}(V)$. Since this argument applies for both players, the abandonment strategies $\left(\gamma_{1}^{*}(V), \gamma_{2}^{*}(V)\right)$, from Equation (42), form a Nash equilibrium. Moreover, player $i$ is only indifferent between any choice of her/his own abandonment intensities exactly when player $j$ uses the abandonment intensity $\gamma_{j}^{*}(V)$, and similarly, player $j$ is only indifferent between any choice of her/his own abandonment intensities exactly when player $i$ uses the abandonment intensity $\gamma_{i}^{*}(V)$, so there is only one Nash equilibrium where the two players use non-trivial abandonment strategies for values of the outcome, $V$ in the interval $\left(A_{M}, A_{Q}\right]$. However, as we formally show in Appendix E, there are exactly three Nash 
equilibria. The two other Nash equilibria are the two pure strategy equilibria corresponding to the cases where one of the players is the follower and the other one is the leader analyzed in sub-sub-section 3.1.1. ${ }^{18}$

Since the two players have the same benefits of becoming a monopolist, $M(V)$, and also have the same rate of on-going investment costs, $k$, it is clear from Equation (42) that the two players choose identical abandonment intensities. We will denote this common abandonment intensity function, $\gamma^{*}(V) .{ }^{19}$ Moreover, the two players will also have identical values of their individual investment projects. We will denote this value $R(V)$. As we have already argued, $R(V)=0$ for all values of the outcome below (and including) $A_{Q}$. For values of the outcome, $V$, above the abandonment threshold level for the follower, $A_{Q}$, in the corresponding leader-follower case, the value of each of the two players' investment projects is a solutions to the same system of ODEs as we set up both for the follower (24) and the leader (34). Hence, the solutions to each of the two ODEs, which we denote $R_{1}(V)$ and $R_{2}(V)$, are ${ }^{20}$

$$
R_{1}(V)=r_{11} V^{z_{1}}+r_{12} V^{z_{2}}-\frac{k T}{2+r T} \quad \text { when } A_{Q} \leq V \leq K,
$$

and

$$
R_{2}(V)=r_{22} V^{z_{2}}+\frac{V}{2+(r-\mu) T}-\frac{k T+K}{2+r T} \quad \text { when } K \leq V .
$$

Moreover, the boundary conditions are

$$
\begin{aligned}
R_{1}\left(A_{Q}\right) & =0, \\
R_{1}^{\prime}\left(A_{Q}\right) & =0, \\
R_{1}(K) & =R_{2}(K),
\end{aligned}
$$

and

$$
R_{1}^{\prime}(K)=R_{2}^{\prime}(K)
$$

Conditions (44a) and (44b) are actually continuity and differentiability conditions at the abandonment threshold level for the follower, $A_{Q}$, in the corresponding leader-follower case. The right hand side of these equations are zero because, as we have argued, $R(V)=0$ for $V \in\left(A_{M}, A_{Q}\right]$ and $R^{\prime}(V)=0$ for $V \in\left(A_{M}, A_{Q}\right) .{ }^{21}$ Conditions (44c) and (44d) are the usual continuity and differentiability conditions at the final investment costs, $K$. The boundary conditions (44) are identical to the boundary conditions for the follower in the corresponding leader-follower case from the system of equations (28). So because both the general solutions to the system of ODEs are the same as for the value of the follower's investment project, compare Equations (27) and (43), and also the boundary conditions are the same, compare Equations (28) and (44), the two players' investment projects have exactly the same value as the value of the follower's

\footnotetext{
${ }_{18}$ Readers familiar with game theory can compare our variable abandonment intensity game with the battle of the sexes game in e.g., Gibbons (1992a,b, Chapter 1). This game also has three Nash equilibria. Two pure strategy equilibria and one (Pareto inferior) mixed strategy equilibrium.

${ }^{19}$ The common value of the investment project in the corresponding monopoly model, $M(V)$, to substitute into Equation (42) is $M_{1}(V)$ from Equation (6a).

${ }^{20}$ To keep the notation consistent with the previous sub-sections of this paper, the subscripts on $R(V)$ now refer to the different general solutions of the different ODEs from the system of ODEs (24) (and (34)) and not to the individual players' values of their individual investment projects.

${ }^{21}$ Since we want $R(V)$ to be differentiable at the value $A_{Q}$, we also have that $R^{\prime}\left(A_{Q}\right)=0$.
} 
investment project in the corresponding leader-follower case. That is, the value of each of the two players' investment projects is

$$
R(V)=Q(V) .
$$

Since the value of each of the two players' investment projects, when they play the variable abandonment intensity game, is the same as the value of the follower's investment project in the corresponding leaderfollower case, the variable abandonment intensity Nash equilibrium is, however, Pareto inferior to both of the two pure strategy leader-follower equilibria. But as we have argued previously, if the players are (truly) identical and they are not allowed to communicate, there is no mechanism to select one of the two leader-follower equilibria from the other. ${ }^{22}$

The values of the follower's investment project (denoted $Q(V)$ ) shown in Figure 6 also represents the values of the two identical players' investment projects when they play the variable abandonment intensity game. There is however an important difference in the investment decisions. The follower abandons her/his investment project as soon as the value of the outcome hits (or is below) the abandonment threshold level, $A_{Q}$. The two players playing the variable abandonment intensity game, on the other hand, keep investing in their individual investment projects also for values of the outcome below the abandonment threshold level, $A_{Q}$. The values of their individual investment projects just happens to be zero for all values of the outcome, $V$ in the range between the abandonment threshold level for the corresponding monopoly model, $A_{M}$, and the abandonment threshold level for the follower in the corresponding leader-follower case, $A_{Q}$, because of the variable abandonment intensity game they are playing with each other in this range. The players apply just the right intensity of abandonment in order to balance the other player's chances of getting the monopoly value and her/his on-going costs of keeping investing. Eventually when one of the two players abandons in this range the value of the other player's investment project jumps up to the corresponding monopoly value (denoted $M(V)$ in Figure 6).

In Figure 7 we show the abandonment intensities for each of the two players as a function of the value of the outcome, $V$, with our base case parameters. We denote the abandonment intensities $\gamma^{*}(V)$. In the figure we also show the abandonment threshold level for the follower in the corresponding leader-follower case and the abandonment threshold level in the corresponding monopoly model on the horizontal axis. We denote the abandonment threshold level for the follower in the corresponding leader-follower case as $A_{Q}$ and the abandonment threshold level in the corresponding monopoly model $A_{M}$. The exact levels are $A_{Q}=6.30$ and $A_{M}=5.26$ with our base case parameters. For values of the outcome below $A_{M}$ the abandonment intensities are infinitely high and for values of the outcome above $A_{Q}$ the abandonment intensities are zero. This means that the abandonment intensity jumps at the abandonment threshold levels for the follower in the corresponding leader-follower case, $A_{Q}$. That is, $\gamma^{*}\left(A_{Q}\right)=3.89$ with our base case parameters. But for any $V>A_{Q}, \gamma^{*}(V)=0$. Finally, note how dramatically the abandonment intensities increase as the value of the outcome, $V$, becomes smaller. This is so because as $V$ becomes smaller so does the value of becoming the monopolist but the on-going costs of keeping investing stays at the same rate, $k$.

\footnotetext{
${ }^{22}$ This is also the case in the battle of the sexes game (Gibbons, 1992a,b, Chapter 1). In this game the mixed strategy equilibrium is also Pareto inferior to the two pure strategy equilibria. But again, if the two players are not allowed to communicate, there is no mechanism to select one of the two pure strategy equilibria from the other.
} 


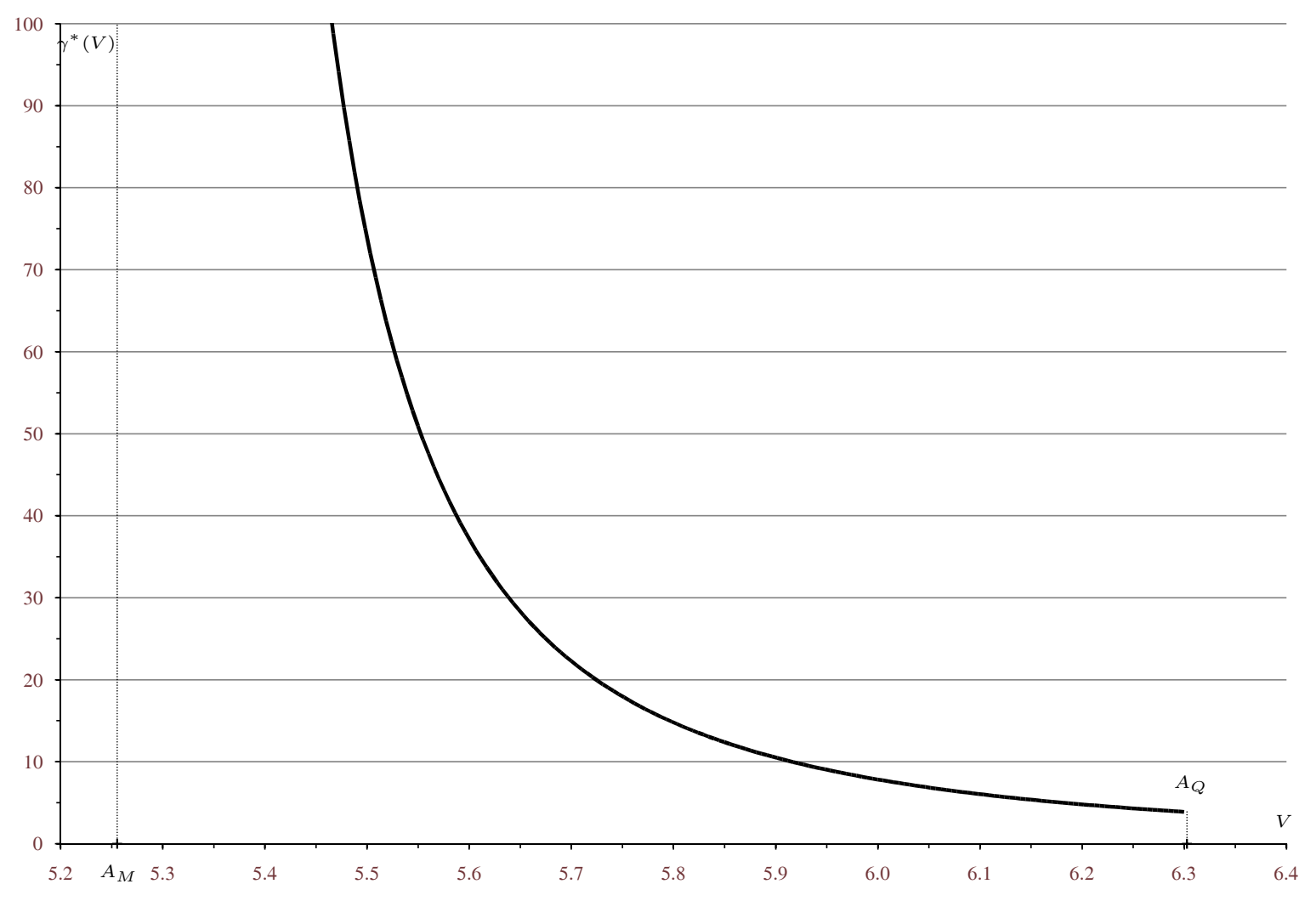

Figure 7. Abandonment intensities (denoted $\gamma^{*}(V)$ ) as a function of the value of the outcome, $V$. The figure also shows the abandonment threshold levels for the follower (denoted $\left.A_{Q}\right)$ in the corresponding leader-follower case and the abandonment threshold level in the corresponding monopoly model (denoted $A_{M}$ ) on the horizontal axis. The exact levels are $A_{Q}=6.30$ and $A_{M}=5.26$. For values of the outcome below $A_{M}$ the abandonment intensities are infinitely high and for values of the outcome above $A_{Q}$ the abandonment intensities are zero. All other parameters are given in Table 1.

\begin{tabular}{c|cc|c|}
\multicolumn{2}{c}{ Active } & \multicolumn{2}{c}{ Player two } \\
\cline { 3 - 4 } Player one & Active & $\lambda(V-K)-2 \lambda U^{1}(V)-k, \lambda(V-K)-2 \lambda U^{2}(V)-k$ & $\lambda(V-K)-\lambda U^{1}(V)-k,-\lambda U^{2}(V)$ \\
\cline { 3 - 4 } & Passive & $-\lambda U^{1}(V), \lambda(V-K)-\lambda U^{2}(V)-k$ & 0,0 \\
\cline { 3 - 4 } & &
\end{tabular}

TABLE 3. Normal form representation of the switching game.

3.2. Model with Switching Option. Finally we will consider the model where two identical players each can switch (costlessly) between an active and a passive investment project. As it was the case in subsection 2.2, where we analyzed the corresponding monopoly model, we find the switching points of the two players by an instantaneous trade-off argument. Again, as it was the case in the corresponding monopoly model, it is clearly optimal for both players to be in passive state when the investment projects are out of the money. Hence, each of the players' switching points will be above the final investment costs, $K$. Let 
$U^{i}(V)$ denote the value of player $i$ 's investment project. The players decide whether to be active or passive by considering the normal form representation of the game in Table 3. Take Player one. The instantaneous benefits of being active is the value flow from the intensity of completion, $\lambda(V-K)$. The instantaneous costs of being active is the intensity of loosing the investment project, which has a value flow of $\lambda U^{1}(V)$, and the on-going investment costs at the rate $k$. If the other player (Player two) is active there is an additional instantaneous cost, namely the intensity of loosing the investment project in case she/he finishes her/his investment project. This instantaneous cost also has a value flow of $\lambda U^{1}(V)$. Player one incurs this flow of costs whether she/he is active or passive her-/himself. We can apply exactly the same arguments for Player two.

Based on the normal form representation of the game in Table 3 we see that Active is a strictly dominating strategy for player $i$ for values of the outcome, $V$, such that

$$
\lambda(V-K)>\lambda U^{i}(V)+k
$$

and that Passive is a strictly dominating strategy for player $i$ for values of the outcome, $V$, such that

$$
\lambda(V-K)<\lambda U^{i}(V)+k .
$$

Hence, if the two players have the same value functions, they will use the same switching point, which we will denote, $S_{U}$. So if the two players are identical, they will use the same switching point and have the same values of their individual investment projects. So we can skip the superscript on $U(V)$ for the values of the two players' investment projects. Moreover, we find the switching point, $S_{U}$, by solving

$$
\lambda\left(S_{U}-K\right)=\lambda U\left(S_{U}\right)+k .
$$

Following arguments similar to the previous models, $U(V)$ must satisfy the system of ODEs

$$
\frac{1}{2} \sigma^{2} V^{2} U^{\prime \prime}(V)+\mu V U^{\prime}(V)-r U(V)=0 \quad \text { when } V<S_{U},
$$

and

$$
\frac{1}{2} \sigma^{2} V^{2} U^{\prime \prime}(V)+\mu V U^{\prime}(V)-(r+2 \lambda) U(V)-k+\lambda(V-K)=0 \quad \text { when } S_{U}<V .
$$

Equation (47a) is the same as Equation (12a) for the corresponding monopoly model and similarly, Equation (47b) is the same as Equation (24b) for the follower and Equation (34b) for the leader. Moreover, the explanations for the terms are the same. Using the same arguments as in the monopoly model, cf. sub-section 2.2, we get the solutions to each of the two ODEs, (47a) and (47b), which we denote $U_{1}(V)$ and $U_{2}(V)$, as

$$
U_{1}(V)=u_{11} V^{x_{1}} \quad \text { when } V \leq S_{U}
$$

and

$$
U_{2}(V)=u_{22} V^{z_{2}}+\frac{V}{2+(r-\mu) T}-\frac{k T+K}{2+r T} \quad \text { when } S_{U} \leq V .
$$

The value of each of the two players' investment projects has the following boundary conditions

$$
U_{1}\left(S_{U}\right)=U_{2}\left(S_{U}\right)
$$


and

$$
U_{1}^{\prime}\left(S_{U}\right)=U_{2}^{\prime}\left(S_{U}\right)
$$

These conditions are the usual continuity and differentiability conditions at the switching point, $S_{U}$. In order to find solutions for the three unknowns, $u_{11}, u_{22}$, and $S_{U}$, we solve the system of equations from (46) and (49). The solution is

$$
\begin{aligned}
S_{U} & =\frac{\left(x_{1}+\left(x_{1}-z_{2}\right)(1+r T)\right)(2+(r-\mu) T)(k T+K)}{\left(x_{1}-1+\left(x_{1}-z_{2}\right)(1+(r-\mu) T)\right)(2+r T)}, \\
u_{11} & =\frac{z_{2}(2+(r-\mu) T)(k T+K)+\left(1-z_{2}\right)(2+r T) S_{U}}{\left(x_{1}-z_{2}\right)(2+(r-\mu) T)(2+r T) S_{U}^{x_{1}}},
\end{aligned}
$$

and

$$
u_{22}=\frac{x_{1}(2+(r-\mu) T)(k T+K)-\left(x_{1}-1\right)(2+r T) S_{U}}{\left(x_{1}-z_{2}\right)(2+(r-\mu) T)(2+r T) S_{U}^{z_{2}}} .
$$

Note the close similarity to the corresponding monopoly solution from the system of equations (20) in subsection 2.2. Note also that by the same arguments as in sub-section 2.2, we can use Equation (46) to argue that $S_{U}>k T+K$ as long as $U\left(S_{U}\right)>0$. Moreover, since $U(V)<N(V)$ for all $V \geq 0$ it is clear that $S_{U}<S_{N}$. Finally, the value of each of the two players' investment project is

$$
U(V)= \begin{cases}u_{11} V^{x_{1}} & \text { when } V<S_{U}, \\ u_{22} V^{z_{2}}+\frac{V}{2+(r-\mu) T}-\frac{k T+K}{2+r T} & \text { when } S_{U} \leq V .\end{cases}
$$

Note that the value function for each of the two players' investment projects, $U(V)$, is not two times differentiable at the duopoly switching point, $S_{U}$, (unless $\lambda=0$ ) because of the way the switching point is selected in the game between the the two players, cf. Equation (46).

If we again look at the special case of the perpetual American option as we did in sub-section 2.2, we see that by inserting $T=0$ into Equation (50a) we get the well-known competitive exercise threshold level for the perpetual American option

$$
S_{U}=K
$$

and thereby a value of zero of the option in this special case. Note that in this special case $U(V)=0$ for all $V$, so it is not a violation of Equation (46) to have $S_{U}=K$.

In Figure 8 we show the values of the two players' investment projects as a function of the value of the outcome, $V$, with our base case parameters. We denote these values $U(V)$. For comparison we also show in the figure the values of the corresponding monopoly investment project and the values of the corresponding investment projects using the coin-toss solution. We denote the values of the corresponding monopoly investment project $N(V)$ and the values of the corresponding investment projects using the cointoss solution $\frac{1}{2}(P+Q)(V)$. (Note that the scale on the horizontal axis in Figure 8 is different from Figure 6 , where we had also shown the coin-toss solution.) In Figure 8 we also show the corresponding switching points on the horizontal axis. The switching point for the duopoly model we denote $S_{U}$ and the switching point for the monopoly model we denote $S_{N}$. The exact levels are $S_{U}=13.05$ and $S_{N}=37.58$ with our base case parameters. For high levels of the outcome the values of the two players' investment projects converge to the value of the corresponding investment project using the coin-toss solution (and thereby also to both 


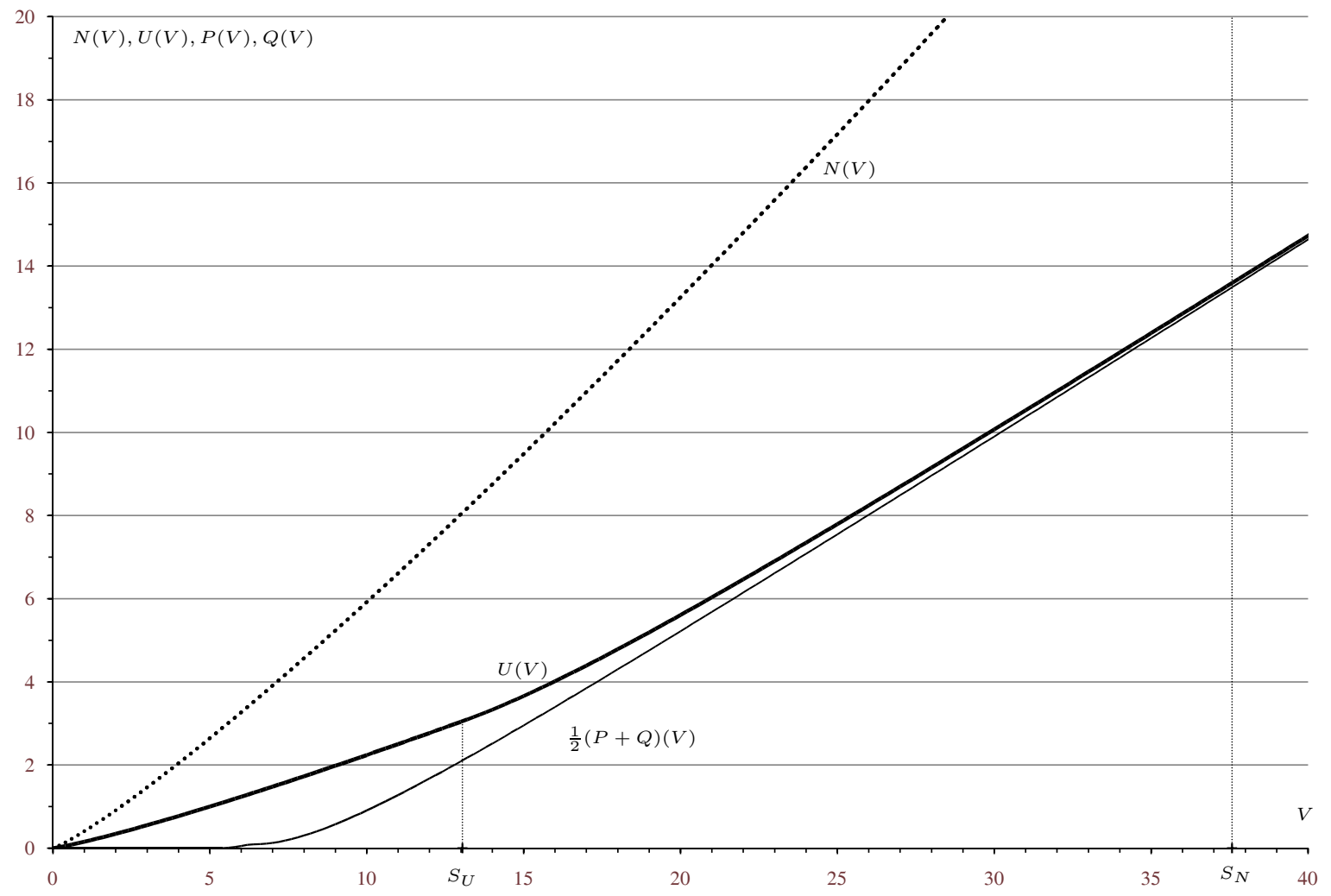

Figure 8. Values of the two players' investment projects (denoted $U(V)$ ) as a function of the value of the outcome, $V$. For comparison the figure also shows the values of the corresponding monopoly investment project (denoted $N(V)$ ) and the values of the corresponding investment projects using the coin-toss solution (denoted $\frac{1}{2}(P+Q)(V)$ ). The figure also shows the corresponding switching points (denoted $S_{U}$ and $S_{N}$ ) on the horizontal axis. The exact levels are $S_{U}=13.05$ and $S_{N}=37.58$. All other parameters are given in Table 1 .

the values of the leader's and the follower's investment projects, cf. Figure 6) because the options to switch and to abandon become far out of the money and thereby their values become relatively small compared to the intrinsic values of the investment projects which are identical for the two players' investment projects and the corresponding investment projects using the coin-toss solution. Finally, a careful look at Figure 8 shows that the curvature of the value function, $U(V)$, changes at the duopoly switching point, $S_{U}$. This indicates that this function is not two times differentiable at the duopoly switching point, $S_{U}$.

In Figure 9 we show the switching points for the duopoly model as a function of the expected time to completion, $T$, with our base case parameters. We denote the switching points for the duopoly model $S_{U}$. For comparison we also show in this figure the corresponding switching point for the monopoly model, cf. sub-section 2.2. We denote the switching points for the monopoly model $S_{N}$. The monopoly switching points are also shown in Figure 4, although in a different scale. The monopoly switching points are substantially higher than the corresponding duopoly switching points for all values of the expected time to completion, $T$, due to the lack of competition. For $T=0$, the switching point in the duopoly model is equal to $K$ (as derived 


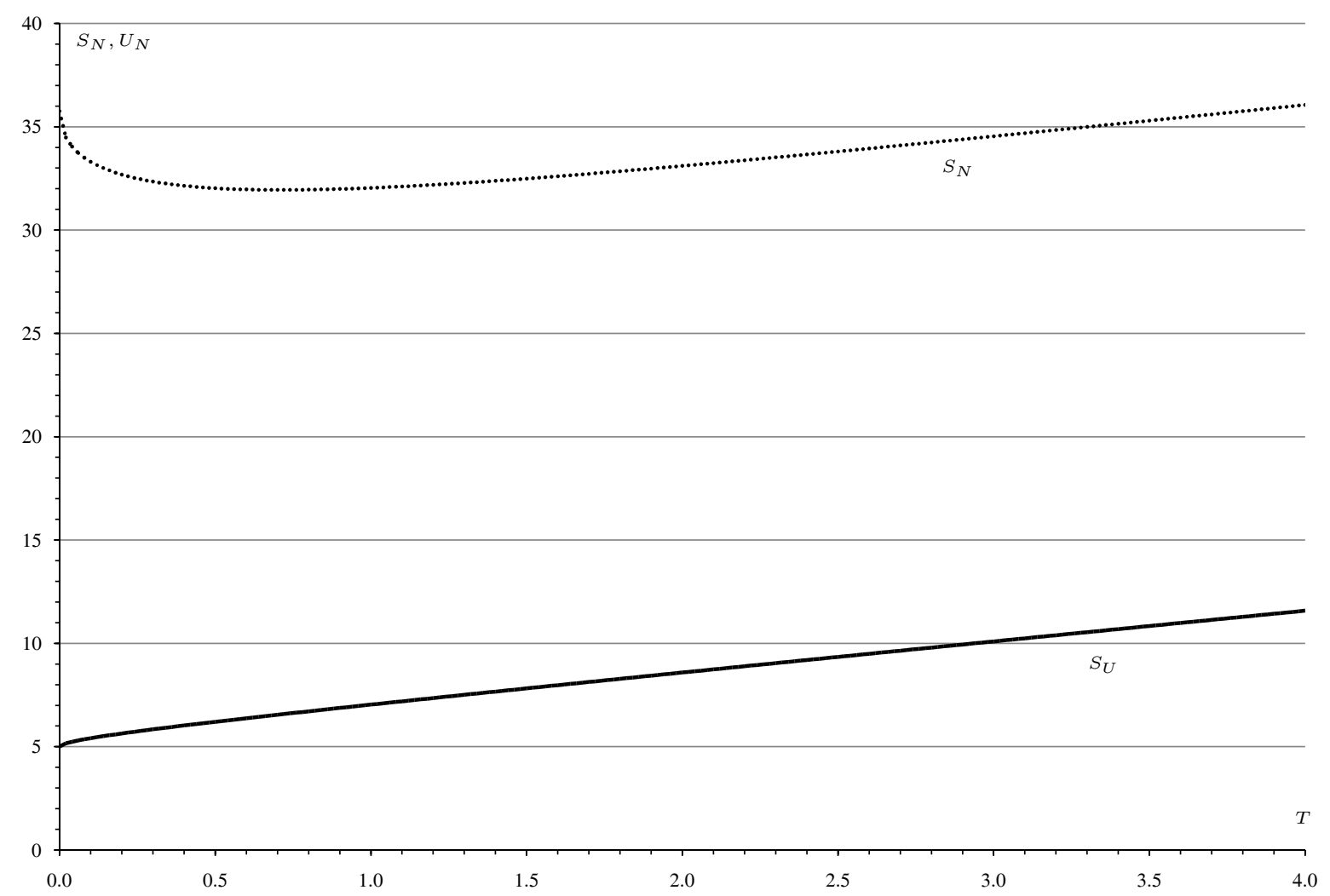

FiguRE 9. Switching points for both the duopoly model (denoted $S_{U}$ ) and the monopoly model (denoted $S_{N}$ ) as a function of expected time to completion, $T$. All other parameters are given in Table 1.

in Equation (52)) and for the monopoly model it is equal to $\frac{x_{1}}{x_{1}-1} K=35.75$ (as derived in Equation (22)) with our base case parameters.

This analysis has some important policy implications. First of all, competition accelerates investments and therefore reduces (expected) time to completion. For values of the outcome between the switching point for the duopolists, $S_{U}$ (which with our base case parameters is around 5-12 depending on the intensity of completion, $\lambda$ ), and the switching point for the monopolist, $S_{N}$ (which with our base case parameters is around 32-36 depending on the intensity of completion, $\lambda$ ), a monopolist would remain passive whereas the duopolists would be active. As we explain in the conclusion (Section 5) we could easily compute the expected time to completion for both of the two cases in closed form and make an exact comparison. Secondly, since the switching point would never go below the final investment costs, $K$, even with a large number of competing investment projects we see that in this model just the step from one monopolist to two duopolists brings us most of the way from monopoly to full competition.

\section{Extensions}

In this section we analyze the model where at the completion of the project the owner obtains a perpetual American option on the value of the outcome. Up to now we have assumed that at the completion date of the 
investment project, the owner must exercise immediately the option to pay the final fixed investment costs. That is, if the project is in the money at the completion date, $\tau$, then the value of the investment project is $V-K$. Moreover, if the investment project happens to be completed at a time when it is out of the money we have up to now assumed that it is valueless. Now we extend the model by giving the owner the option to delay paying the fixed final investment costs in order to obtain the value of the outcome. This extension is especially interesting when the project is completed out of the money. Take, e.g, an R\&D investment or a mine exploration project where a product/mine has been discovered (i.e., we are at the completion date), but the owner would like to wait until the optimal time to build the plant and launch the product/mineral.

We will only develop this extension for the monopoly model with the abandonment option, which we analyzed in sub-section 2.1. Similar analysis could be done for all the other models we have developed in this paper. Instead of the exercise value of the investment project, $\max \{V-K, 0\}$, at the completion date, $\tau$, the owner gets the value of a perpetual American call option. We denote the value of the perpetual American call option $H(V)$. Since the owner is paying on-going investment costs at the rate $k$, there will still be an abandonment threshold level, which we denote $A_{L}$, so that the owner abandons her/his investment project when the value of the outcome is below $A_{L}$. However, since $H(V)$ is always greater than (or equal to) the exercise value of the American call option, $\max \{V-K, 0\}$, it is clear that the abandonment threshold level, $A_{L}$, for this extension is less than the corresponding abandonment threshold level, $A_{M}$, for the monopoly model with abandonment analyzed in sub-section 2.1. Let $L(V)$ denote the value of the investment project for this extension. $L(V)$ must satisfy the ODE

$$
\frac{1}{2} \sigma^{2} V^{2} L^{\prime \prime}(V)+\mu V L^{\prime}(V)-(r+\lambda) L(V)-k+\lambda H(V)=0 \quad \text { when } A_{L}<V .
$$

If we compare the ODE (53) to the system of ODEs (3) for the value of the investment project in subsection 2.1, we have simply changed the completion value from $\max \{V-K, 0\}$ in the system of ODEs (3) to the value of a perpetual American call option, $H(V)$, in the ODE (53). It is well-known that the value of the American perpetual call option, $H(V)$, is

$$
H(V)= \begin{cases}h_{11} V^{x_{1}} & \text { when } V<E_{H}, \\ V-K & \text { when } E_{H} \leq V,\end{cases}
$$

where the optimal exercise threshold level, $E_{H}$, is

$$
E_{H}=\frac{x_{1}}{x_{1}-1} K
$$

and the coefficient in the value function, $h_{11}$, is

$$
h_{11}=\frac{1}{x_{1} E_{H}^{x_{1}-1}} .
$$

Substituting the value of the American perpetual call option from Equation (54) into the ODE (53), and using the boundary conditions for value matching and smooth pasting at the abandonment threshold level $A_{L}$ and continuity and differentiability at the exercise threshold level for the American call option $E_{H}$, we 
get the value of the investment project as ${ }^{23}$

$$
L(V)= \begin{cases}0 & \text { when } V<A_{L}, \\ l_{11} V^{y_{1}}+l_{12} V^{y_{2}}-\frac{k T}{1+r T}+\frac{h_{11} V^{x_{1}}}{1+\left(r-x_{1} \mu-\frac{1}{2} x_{1}\left(x_{1}-1\right) \sigma^{2}\right) T} & \text { when } A_{L} \leq V<E_{H}, \\ l_{22} V^{y_{2}}+\frac{V}{1+(r-\mu) T}-\frac{k T+K}{1+r T} & \text { when } E_{H} \leq V .\end{cases}
$$

Here the coefficients, $l_{11}, l_{12}$, and $l_{22}$, are

$$
\begin{aligned}
& l_{11}=\frac{\frac{y_{2} K}{(1+r T) E_{H}}+\frac{1-y_{2}}{1+(r-\mu) T}-\frac{\left(x_{1}-y_{2}\right) h_{11}}{\left(1+\left(r-x_{1} \mu-\frac{1}{2} x_{1}\left(x_{1}-1\right) \sigma^{2}\right) T\right) E_{H}^{x_{1}-1}}}{\left(y_{1}-y_{2}\right) E_{H}^{y_{1}-1}}, \\
& l_{12}=-\frac{y_{1} h_{11} A_{L}^{y_{1}}+\frac{x_{1} h_{11} A_{L}^{x_{1}}}{1+\left(r-x_{1} \mu-\frac{1}{2} x_{1}\left(x_{1}-1\right) \sigma^{2}\right) T}}{y_{2} A_{L}^{y_{2}}}
\end{aligned}
$$

and

$$
l_{22}=l_{12}+\left(l_{11} E_{H}^{y_{1}}+\frac{K}{1+r T}-\frac{E_{H}}{1+(r-\mu) T}+\frac{h_{11} E_{H}^{x_{1}}}{1+\left(r-x_{1} \mu-\frac{1}{2} x_{1}\left(x_{1}-1\right) \sigma^{2}\right) T}\right) \frac{1}{E_{H}^{y_{2}}}
$$

In order to find the abandonment threshold level, $A_{L}$, we need to solve the remaining boundary condition,

$$
L\left(A_{L}\right)=0,
$$

numerically using the closed form solutions from the system of equations (58).

Figure 10 shows that the value in this case, $L(V)$, is always larger than the monopoly case we discussed in sub-section 2.1, $M(V)$, especially for lower values of the outcome, $V$. For very high values of the outcome these two values will converge since the completion will bring immediate exercise of the American call option. The figure also shows the completion values for these two cases, $H(V)$ and $V-K$.

\section{Conclusions}

In this paper we develop a simple, yet powerful, framework to deal with real investment projects with uncertain investment costs and time to completion. We apply the framework to situations in which the owner of the project has monopoly rights to the outcome of the project, and to situations in which there are two owners who simultaneously invest, but where only one of them (the first to complete) may obtain the rights to the outcome. We obtain analytical solutions to important cases with abandonment and/or switching to different levels of investment activity. Hence, we are able to easily compare the project values and abandonment/switching threshold levels for the different cases.

Our framework can also be applied to derive closed form solutions to the expected time to completion, probability of completion, expected cost until completion, etc. Basically, all of these key values satisfy very similar ODEs as the value of the investment project. One simply adjusts the payoff rates and the discount rates in the ODEs. Hence, the formulas for these key values have the same structure as the formula for the

\footnotetext{
${ }^{23}$ As in the other models with abandonment options we have analyzed in this paper, there are different situations. Here we analyze the most common (and also most complicated) situation when $A_{L}<E_{H}$. However, even though it may seem quite exotic, we cannot rule out that for extremely high on-going costs, $k$, the abandonment threshold level for the investment project, $A_{L}$, is so high that it is above the exercise threshold level, $E_{H}$, for the American option received at completion. In this situation the value of the investment project is identical to the standard monopoly model analyzed in sub-section 2.1 . That is, if $\tilde{A_{M}}$ from Equation (11a) is greater than (or equal to) $E_{H}$ from Equation (55), then the value of the investment project- even with the American option extension-is still given by Equation (10) in sub-section 2.1.
} 




FiguRE 10. Values of the extended investment project (denoted $L(V)$ ) as a function of the value of the outcome, $V$, and its corresponding abandonment threshold level (denoted $A_{L}$ ). The figure also shows completion values of the investment project (denoted $H(V)$ ) as a function of the value of the outcome and its corresponding exercise threshold level (denoted $E_{H}$ ). The exact levels are $A_{L}=3.79$, and $E_{H}=35.75$. For comparison the figure also shows the value of the (standard) investment project with the abandonment option (denoted $M(V)$ ) and its corresponding abandonment threshold level (denoted $A_{M}$ ). The exact level is $A_{M}=5.25$. Finally, the figure also shows the completion values of the (standard) investment project (denoted $V-K$ ) as a function of the value of the outcome. All other parameters are given in Table 1.

value of the investment projects. Moreover, the value of the investment project can also be divided into three parts: the value of the outcome at completion, the value of all the on-going investment costs until completion (or abandonment), and the value of the fixed investment costs at completion. However, it is always necessary first to solve for the value of the project in order to find the optimal abandonment/switching levels.

The duopoly models we have developed easily extend to more than two players. For example in the abandonment case, the leader-follower solution easily extends to more than two players if we know the order in which they abandon. Given this solution, the variable abandonment intensity solution also easily extends to more than two players. 
Anther interesting extension of our framework would be to include asymmetric information between the two players. For example, each player knows her/his own on-going investment cost rate exactly, but she/he only knows the distribution of the other player's on-going costs. The knowledge of the other player's on-going investment costs is updated as time progresses observing that he/she still has not abandoned.

Finally, a possible application of our framework is to analyze the design of research incentives for nonprofitable R\&D investments as analyzed numerically by Hsu and Schwartz (2006).

\section{Appendix A. Monopoly Model with Abandonment Option: Derivation of the value of the inVESTMEnt PRoJect When $A_{M} \geq K$.}

Assume that the abandonment threshold level is above the final investment costs, $K$. In this situation, the value of the investment project (still denoted $M(V)$ ) must satisfy the ODE

$$
\frac{1}{2} \sigma^{2} V^{2} M^{\prime \prime}(V)+\mu V M^{\prime}(V)-(r+\lambda) M(V)-k+\lambda(V-K)=0 \quad \text { when } \tilde{A}_{M}<V .
$$

Note that we denote the abandonment threshold level $\tilde{A}_{M}$ to emphasize that it may be different from the solution obtained in Equation (8b). The general solution of the ODE (60), which we denote $M_{3}(V)$, is

$$
M_{3}(V)=m_{32} V^{y_{2}}+\frac{V}{1+(r-\mu) T}-\frac{k T+K}{1+r T} \quad \text { when } \tilde{A}_{M} \leq V .
$$

Using the same arguments as in sub-section 2.1, we have eliminated the $V^{y_{1}}$ term in Equation (61). The boundary conditions are

$$
M_{3}\left(\tilde{A}_{M}\right)=0
$$

and

$$
M_{3}^{\prime}\left(\tilde{A}_{M}\right)=0 .
$$

In this situation we just solve the system of equations (62) in the two unknowns $m_{32}$ and $\tilde{A}_{M}$. The solution is

$$
\tilde{A}_{M}=\frac{y_{2}(1+(r-\mu) T)(k T+K)}{\left(y_{2}-1\right)(1+r T)}
$$

and

$$
m_{32}=-\frac{\tilde{A}_{M}^{1-y_{2}}}{y_{2}(1+(r-\mu) T)} .
$$

Finally, the value of the investment project is

$$
M(V)= \begin{cases}0 & \text { when } V<\tilde{A}_{M}, \\ m_{32} V^{y_{2}}+\frac{V}{1+(r-\mu) T}-\frac{k T+K}{1+r T} & \text { when } \tilde{A}_{M} \leq V .\end{cases}
$$

\section{Appendix B. Derivation of the Monopoly Model with both Abandonment and Switching} Options

As in the first model we analyzed in sub-section 2.1 we will have to consider both the situation where we assume that the abandonment threshold level, $A_{O}$, is below the final investment costs, $K$, and the situation 
where it is above. So first we assume that $A_{O}<K$. Let $O(V)$ denote the value of the investment project for this model. $O(V)$ must satisfy the system of ODEs

$$
\begin{array}{ll}
\frac{1}{2} \sigma^{2} V^{2} O^{\prime \prime}(V)+\mu V O^{\prime}(V)-(r+\lambda) O(V)-k=0 & \text { when } A_{O}<V<K, \\
\frac{1}{2} \sigma^{2} V^{2} O^{\prime \prime}(V)+\mu V O^{\prime}(V)-(r+\lambda) O(V)-k+\lambda(V-K)=0 & \text { when } K<V<S_{O},
\end{array}
$$

and

$$
\frac{1}{2} \sigma^{2} V^{2} O^{\prime \prime}(V)+\mu V O^{\prime}(V)-(r+\bar{\lambda}) O(V)-\bar{k}+\bar{\lambda}(V-K)=0 \quad \text { when } S_{O}<V .
$$

The general solutions to each of the three ODEs, (63a), (63b), and (63c), which we denote $O_{1}(V), O_{2}(V)$, and $O_{3}(V)$, are

$$
\begin{array}{ll}
O_{1}(V)=o_{11} V^{y_{1}}+o_{12} V^{y_{2}}-\frac{k T}{1+r T} & \text { when } A_{O} \leq V \leq K, \\
O_{2}(V)=o_{21} V^{y_{1}}+o_{22} V^{y_{2}}+\frac{V}{1+(r-\mu) T}-\frac{k T+K}{1+r T} & \text { when } K \leq V \leq S_{O},
\end{array}
$$

and

$$
O_{3}(V)=o_{31} V^{w_{1}}+o_{32} V^{w_{2}}+\frac{V}{1+(r-\mu) \bar{T}}-\frac{\bar{k} \bar{T}+K}{1+r \bar{T}} \quad \text { when } S_{O} \leq V .
$$

Here the powers, $w_{1}$ and $w_{2}$, are given by

$$
w_{1}=\frac{\left(\frac{1}{2} \sigma^{2}-\mu\right)+\sqrt{\left(\mu-\frac{1}{2} \sigma^{2}\right)^{2}+2(r+\bar{\lambda}) \sigma^{2}}}{\sigma^{2}}>y_{1}>1
$$

and

$$
w_{2}=\frac{\left(\frac{1}{2} \sigma^{2}-\mu\right)-\sqrt{\left(\mu-\frac{1}{2} \sigma^{2}\right)^{2}+2(r+\bar{\lambda}) \sigma^{2}}}{\sigma^{2}}<y_{2}<0 .
$$

The powers $y_{1}$ and $y_{2}$ are still defined by the system of equations (5). Comparing the powers $w_{1}$ and $w_{2}$ with the corresponding powers $y_{1}$ and $y_{2}$, we see that $w_{1}>y_{1}>1$ and that $w_{2}<y_{2}<0$. Using the same arguments as for the previous models, we know that $\lim _{V \rightarrow \infty} \frac{O_{3}(V)}{V}$ must be finite. Hence, we can eliminate the $V^{w_{1}}$ term in Equation (64c). The value of the investment project has the following boundary conditions

$$
\begin{aligned}
O_{1}\left(A_{O}\right) & =0, \\
O_{1}^{\prime}\left(A_{O}\right) & =0, \\
O_{1}(K) & =O_{2}(K), \\
O_{1}^{\prime}(K) & =O_{2}^{\prime}(K), \\
O_{2}\left(S_{O}\right) & =O_{3}\left(S_{O}\right),
\end{aligned}
$$

and

$$
O_{2}^{\prime}\left(S_{O}\right)=O_{3}^{\prime}\left(S_{O}\right)
$$


Condition (66a) reflects the fact that at the abandonment threshold level, $A_{O}$, the value of the investment project is zero. Condition (66b) is the usual smooth pasting condition at the abandonment threshold level. Conditions (66c) and (66d) are the usual continuity and differentiability conditions at the final investment costs, $K$, and similarly, Conditions (66e) and (66f) are the usual continuity and differentiability conditions at the switching point, $S_{O}$. In order to find the optimal switching point, $S_{O}$, we solve

$$
(\bar{\lambda}-\lambda)\left(S_{O}-K\right)=(\bar{\lambda}-\lambda) O_{3}\left(S_{O}\right)+(\bar{k}-k) .
$$

Equation (66g) is based on the following instantaneous trade-off argument. At each instant in time the owner considers instantaneous costs and benefits from switching between the two activity levels. The increased instantaneous benefits from switching from the low to the high activity level is the increased intensity of completion which has a value flow $(\bar{\lambda}-\lambda)(V-K)$ per unit of time. On the other hand the increased instantaneous costs of switching are (i) the increased intensity of loosing the investment project which has a value flow $(\bar{\lambda}-\lambda) O_{3}(V)$ per unit of time and (ii) the increased on-going investment costs at the rate $\bar{k}-k$ per unit of time. By the same arguments as in the switching model from sub-section 2.2, Equation (66g) also implies that the value function, $O(V)$, will be two times differentiable at the switching point, $S_{O}$. That is,

$$
O_{2}^{\prime \prime}\left(S_{O}\right)=O_{3}^{\prime \prime}\left(S_{O}\right)
$$

In order to find solutions for the seven unknowns, $o_{11}, o_{12}, o_{21}, o_{22}, o_{32}, A_{O}$, and $S_{O}$, we must solve the system of equations (66). We are able to solve five of these equations in closed form as functions of $A_{O}$ and $S_{O}$ using the Equations (66a), (66b), (66c), (66d), and (66f):

$$
\begin{aligned}
& o_{11}=-\frac{y_{2} k T}{\left(y_{1}-y_{2}\right)(1+r T) A_{O}^{y_{1}}}, \\
& o_{12}=\frac{y_{1} k T}{\left(y_{1}-y_{2}\right)(1+r T) A_{O}^{y_{2}}}, \\
& o_{21}=o_{11}-\frac{1+\left(r-y_{2} \mu\right) T}{\left(y_{1}-y_{2}\right)(1+(r-\mu) T)(1+r T) K^{y_{1}-1}}, \\
& o_{22}=o_{12}+\frac{\left(1+\left(r-y_{1} \mu\right) T\right) K^{1-y_{2}}}{\left(y_{1}-y_{2}\right)(1+(r-\mu) T)(1+r T)},
\end{aligned}
$$

and

$$
o_{32}=\left(y_{1} o_{21} S_{O}^{y_{1}-1}+y_{2} o_{22} S_{O}^{y_{2}-1}+\frac{1}{1+(r-\mu) T}-\frac{1}{1+(r-\mu) \bar{T}}\right) \frac{S_{O}^{1-w_{2}}}{w_{2}} .
$$

In order to find $A_{O}$ and $S_{O}$, we need to solve the two remaining equations, (66e) and (66g), numerically using the closed form solutions from the system of equations (68). Finally, the value of the investment project is

$$
O(V)= \begin{cases}0 & \text { when } V<A_{O}, \\ o_{11} V^{y_{1}}+o_{12} V^{y_{2}}-\frac{k T}{1+r T} & \text { when } A_{O} \leq V<K, \\ o_{21} V^{y_{1}}+o_{22} V^{y_{2}}+\frac{V}{1+(r-\mu) T}-\frac{k T+K}{1+r T} & \text { when } K \leq V<S_{O}, \\ o_{32} V^{w_{2}}+\frac{V}{1+(r-\mu) T}-\frac{\bar{k} \bar{T}+K}{1+r T} & \text { when } S_{O} \leq V .\end{cases}
$$


If it turns out that the numerical solution to $A_{O}$ is greater than $K$, we reconsider the problem. In this situation, the value of the investment project (still denoted $O(V)$ ) must satisfy the system of ODEs

$$
\frac{1}{2} \sigma^{2} V^{2} O^{\prime \prime}(V)+\mu V O^{\prime}(V)-(r+\lambda) O(V)-k+\lambda(V-K)=0 \quad \text { when } \tilde{A}_{O}<V<\tilde{S}_{O}
$$

and

$$
\frac{1}{2} \sigma^{2} V^{2} O^{\prime \prime}(V)+\mu V O^{\prime}(V)-(r+\bar{\lambda}) O(V)-\bar{k}+\bar{\lambda}(V-K)=0 \quad \text { when } \tilde{S}_{O}<V .
$$

Note that we denote the abandonment threshold level $\tilde{A}_{O}$ and the switching point $\tilde{S}_{O}$ to emphasize that they may be different from the (numerical) solutions obtained above. The general solutions to each of the two ODEs, (70a) and (70b), which we denote $O_{4}(V)$ and $O_{5}(V)$, are

$$
O_{4}(V)=o_{41} V^{y_{1}}+o_{42} V^{y_{2}}+\frac{V}{1+(r-\mu) T}-\frac{k T+K}{1+r T} \quad \text { when } \tilde{A}_{O} \leq V \leq \tilde{S}_{O}
$$

and

$$
O_{5}(V)=o_{52} V^{w_{2}}+\frac{V}{1+(r-\mu) \bar{T}}-\frac{\bar{k} \bar{T}+K}{1+r \bar{T}} \quad \text { when } \tilde{S}_{O} \leq V .
$$

Using the same arguments as in the previous models, we have eliminated the $V^{w_{1}}$ term in Equation (71b). The boundary conditions are

$$
\begin{aligned}
& O_{4}\left(\tilde{A}_{O}\right)=0, \\
& O_{4}^{\prime}\left(\tilde{A}_{O}\right)=0, \\
& O_{4}\left(\tilde{S}_{O}\right)=O_{5}\left(\tilde{S}_{O}\right),
\end{aligned}
$$

and

$$
O_{4}^{\prime}\left(\tilde{S}_{O}\right)=O_{5}^{\prime}\left(\tilde{S}_{O}\right)
$$

In order to find the optimal switching point, $\tilde{S}_{O}$, we solve

$$
(\bar{\lambda}-\lambda)\left(\tilde{S}_{O}-K\right)=(\bar{\lambda}-\lambda) O_{5}\left(\tilde{S}_{O}\right)+(\bar{k}-k) .
$$

In order to find solutions for the five unknowns, $o_{41}, o_{42}, o_{52}, \tilde{A}_{O}$, and $\tilde{S}_{O}$, we must solve the system of equations (72). We are able to solve three of these equations in closed form as functions of $\tilde{A}_{O}$ and $\tilde{S}_{O}$ using the Equations $(72 \mathrm{a}),(72 \mathrm{~b})$, and $(72 \mathrm{~d})$ :

$$
\begin{aligned}
& o_{41}=-\frac{\left(1-y_{2}\right)(1+r T) \tilde{A}_{O}+y_{2}(1+(r-\mu) T)(k T+K)}{\left(y_{1}-y_{2}\right)(1+(r-\mu) T)(1+r T) \tilde{A}_{O}^{y_{1}}}, \\
& o_{42}=-\frac{\left(y_{1}-1\right)(1+r T) \tilde{A}_{O}-y_{1}(1+(r-\mu) T)(k T+K)}{\left(y_{1}-y_{2}\right)(1+(r-\mu) T)(1+r T) \tilde{A}_{O}^{y_{2}}},
\end{aligned}
$$

and

$$
o_{52}=\left(y_{1} o_{41} \tilde{S}_{O}^{y_{1}-1}+y_{2} o_{42} \tilde{S}_{O}^{y_{2}-1}+\frac{1}{1+(r-\mu) T}-\frac{1}{1+(r-\mu) \bar{T}}\right) \frac{\tilde{S}_{O}^{1-w_{2}}}{w_{2}}
$$


In order to find $\tilde{A}_{O}$ and $\tilde{S}_{O}$, we need to solve the two remaining equations, (72c) and (72e), numerically using the closed form solutions from the system of equations (73). Finally, the value of the investment project is

$$
O(V)= \begin{cases}0 & \text { when } V<\tilde{A}_{O}, \\ o_{41} V^{y_{1}}+o_{42} V^{y_{2}}+\frac{V}{1+(r-\mu) T}-\frac{k T+K}{1+r T} & \text { when } \tilde{A}_{O} \leq V<\tilde{S}_{O}, \\ o_{52} V^{w_{2}}+\frac{V}{1+(r-\mu) T}-\frac{\bar{k} \bar{T}+K}{1+r T} & \text { when } \tilde{S}_{O} \leq V .\end{cases}
$$

Appendix C. Duopoly Model with Abandonment Option: Derivation of the value of the FOLLOWER'S INVESTMENT PROJECT WHEN $A_{Q} \geq K$.

Assume that the abandonment threshold level for the follower is above the final investment costs, $K$. In this situation, the value of the investment project (still denoted $Q(V)$ ) must satisfy the ODE

$$
\frac{1}{2} \sigma^{2} V^{2} Q^{\prime \prime}(V)+\mu V Q^{\prime}(V)-(r+2 \lambda) Q(V)-k+\lambda(V-K)=0 \quad \text { when } \tilde{A}_{Q}<V .
$$

Note that we denote the abandonment threshold level for the follower $\tilde{A}_{Q}$ to emphasize that it may be different from the solution obtained above in Equation (29b). The general solution to the ODE (75), which we denote $Q_{3}(V)$, is

$$
Q_{3}(V)=q_{32} V^{z_{2}}+\frac{V}{2+(r-\mu) T}-\frac{k T+K}{2+r T} \quad \text { when } \tilde{A}_{Q} \leq V .
$$

Using the same arguments as in the previous models, we have eliminated the $V^{z_{1}}$ term in Equation (76). The boundary conditions are

$$
Q_{3}\left(\tilde{A}_{Q}\right)=0
$$

and

$$
Q_{3}^{\prime}\left(\tilde{A}_{Q}\right)=0 .
$$

In this situation we just solve the system of equations (77) in the two unknowns $q_{32}$ and $\tilde{A}_{Q}$. The solution is

$$
\tilde{A}_{Q}=\frac{z_{2}(2+(r-\mu) T)(k T+K)}{\left(z_{2}-1\right)(2+r T)}
$$

and

$$
q_{32}=-\frac{\tilde{A}_{Q}^{1-z_{2}}}{z_{2}(2+(r-\mu) T)} .
$$

Finally, the value of the follower's investment project is

$$
Q(V)= \begin{cases}0 & \text { when } V<\tilde{A}_{Q}, \\ q_{32} V^{z_{2}}+\frac{V}{2+(r-\mu) T}-\frac{k T+K}{2+r T} & \text { when } \tilde{A}_{Q} \leq V .\end{cases}
$$

Appendix D. Duopoly Model with Abandonment Option: Derivation of the value of the LEADER'S INVESTMENT PROJECT WHEN $A_{Q} \geq K$.

Assume that the abandonment threshold level for the follower is above the final investment costs, $K$. For values of the outcome, $V$, above the abandonment threshold level for the follower, $\tilde{A}_{Q}$, the value of the 
leader's investment project (still denoted $P(V)$ ) must satisfy the ODE

$$
\frac{1}{2} \sigma^{2} V^{2} P^{\prime \prime}(V)+\mu V P^{\prime}(V)-(r+2 \lambda) P(V)-k+\lambda(V-K)=0 \quad \text { when } \tilde{A}_{Q}<V .
$$

The value of the leader's investment project is equal to the value of the corresponding investment project in the monopoly model as soon as the follower has abandoned so in this situation we have

$$
P(V)=M(V) \quad \text { when } A_{M} \leq V \leq \tilde{A}_{Q} .
$$

The general solution to the ODE (78), which we denote $P_{3}(V)$, is

$$
P_{3}(V)=p_{32} V^{z_{2}}+\frac{V}{2+(r-\mu) T}-\frac{k T+K}{2+r T} \quad \text { when } \tilde{A}_{Q} \leq V .
$$

Using the same arguments as in the previous models, we have (again) eliminated the $V^{z_{1}}$ term in Equation $(79 \mathrm{~b})$. The boundary condition is

$$
P_{3}\left(\tilde{A}_{Q}\right)=M\left(\tilde{A}_{Q}\right)
$$

In this situation we just solve Equation (80) in the unknown $p_{32}$. The solution is ${ }^{24}$

$$
p_{32}= \begin{cases}\left(m_{22} \tilde{A}_{Q}^{y_{2}}-\frac{k T+K}{(1+r T)(2+r T)}+\frac{\tilde{A}_{Q}}{(1+(r-\mu) T)(2+(r-\mu) T)}\right) \frac{1}{\tilde{A}_{Q}^{z_{2}}} & \text { when } A_{M}<K \\ \left(m_{32} \tilde{A}_{Q}^{y_{2}}-\frac{k T+K}{(1+r T)(2+r T)}+\frac{\tilde{A}_{Q}}{(1+(r-\mu) T)(2+(r-\mu) T)}\right) \frac{1}{\tilde{A}_{Q}^{z_{2}}} & \text { when } A_{M} \geq K .\end{cases}
$$

Finally, the value of the leader's investment project is

$$
P(V)= \begin{cases}0 & \text { when } V<A_{M}, \\ M(V) & \text { when } A_{M} \leq V<\tilde{A}_{Q}, \\ p_{32} V^{z_{2}}+\frac{V}{2+(r-\mu) T}-\frac{k T+K}{2+r T} & \text { when } \tilde{A}_{Q} \leq V .\end{cases}
$$

For completeness we will substitute $M(V)$ into both Equation (38) and Equation (82). If $A_{Q}<K$ then also $A_{M}<K$. In this situation we get $P(V)$ from Equation (38) and $M(V)$ from Equation (9). The solution to $P(V)$ expands to

$$
P(V)= \begin{cases}0 & \text { when } V<A_{M}, \\ m_{11} V^{y_{1}}+m_{12} V^{y_{2}}-\frac{k T}{1+r T} & \text { when } A_{M} \leq V<A_{Q}, \\ p_{11} V^{z_{1}}+p_{12} V^{z_{2}}-\frac{k T}{2+r T} & \text { when } A_{Q} \leq V<K, \\ p_{22} V^{z_{2}}+\frac{V}{2+(r-\mu) T}-\frac{k T+K}{2+r T} & \text { when } K \leq V .\end{cases}
$$

If $\tilde{A}_{Q} \geq K$ and $A_{M}<K$ then we get $P(V)$ from Equation (82) and $M(V)$ from Equation (9). In this situation the solution to $P(V)$ expands to

$$
P(V)= \begin{cases}0 & \text { when } V<A_{M}, \\ m_{11} V^{y_{1}}+m_{12} V^{y_{2}}-\frac{k T}{1+r T} & \text { when } A_{M} \leq V<K, \\ m_{22} V^{y_{2}}+\frac{V}{1+(r-\mu) T}-\frac{k T+K}{1+r T} & \text { when } K \leq V<\tilde{A}_{Q}, \\ p_{32} V^{z_{2}}+\frac{V}{2+(r-\mu) T}-\frac{k T+K}{2+r T} & \text { when } \tilde{A}_{Q} \leq V .\end{cases}
$$

\footnotetext{
${ }^{24}$ Note that $M(V)$ on the right hand side of Equation (80) can be either $M_{2}(V)$ from Equation (6b) or $M_{3}(V)$ from Equation (61) (in Appendix A) depending on whether $A_{M}<K$ or $\tilde{A}_{M} \geq K$ (which is equivalent to $A_{M} \geq K$, cf. footnote 7). Therefore we will either have an $m_{22}$ or an $m_{32}$ term in the solution to $p_{32}$ in Equation (81).
} 
Finally, if $\tilde{A}_{Q} \geq K$ and $A_{M} \geq K$ then we get $P(V)$ from Equation (82) and $M(V)$ from Equation (10). In this situation the abandonment threshold level in the corresponding monopoly model is $\tilde{A}_{M}$ from Equation (11a) and the solution to $P(V)$ expands to

$$
P(V)= \begin{cases}0 & \text { when } V<\tilde{A}_{M} \\ m_{32} V^{y_{2}}+\frac{V}{1+(r-\mu) T}-\frac{k T+K}{1+r T} & \text { when } \tilde{A}_{M} \leq V<\tilde{A}_{Q} \\ p_{32} V^{z_{2}}+\frac{V}{2+(r-\mu) T}-\frac{k T+K}{2+r T} & \text { when } \tilde{A}_{Q} \leq V\end{cases}
$$

\section{Appendix E. Proof that the Variable Abandonment Intensities Solution is a Nash} EQUILIBRIUM.

To prove that the abandonment strategies $\left(\gamma_{1}^{*}(V), \gamma_{2}^{*}(V)\right)$, from Equation (42), form a Nash equilibrium, we look at each of the players best response correspondences. Player $i$ 's best response correspondence is player $i$ 's set of best responses ${ }^{25}$ (in form of a set of abandonment intensities) to any given abandonment intensity, $\gamma_{j}$, chosen by player $j$, for a given value of the outcome, $V$. We denote player $i$ 's best response correspondence $\gamma_{i}^{*}\left(\gamma_{j} ; V\right)$.

In order to find player $i$ 's (set of) best responses to any given strategy, $\gamma_{j}$, chosen by player $j$, we study player $i$ 's instantaneous benefits and costs for a given value of the outcome, $V$, in the interval $\left(A_{M}, A_{Q}\right]$, and for given strategies chosen by the two players, $\gamma_{i}$ and $\gamma_{j}$. Player $i$ 's instantaneous benefits are the intensity of becoming the monopolist, which only happens if player $j$ abandons. This has a value flow $\gamma_{j} M_{i}(V)$ per unit of time. (There is no value flow from the intensity of completion since player $i$ 's investment project is out of the money.) Player $i$ 's instantaneous costs are (i) the intensity of loosing the investment project either due to abandonment from one of the players or due to completion (out of the money), which has a value flow $\left(2 \lambda+\gamma_{i}+\gamma_{j}\right) R_{i}(V)$ per unit of time, and (ii) the on-going costs at the rate $k_{i}$ per unit of time. Hence, player $i$ 's instantaneous net benefits (denoted $\pi_{i}\left(\gamma_{i}, \gamma_{j} ; V\right)$ ) are

$$
\pi_{i}\left(\gamma_{i}, \gamma_{j} ; V\right)=\gamma_{j} M_{i}(V)-\left(2 \lambda+\gamma_{i}+\gamma_{j}\right) R_{i}(V)-k_{i} .
$$

Since we know that $R_{i}(V)=0, \pi_{i}\left(\gamma_{i}, \gamma_{j} ; V\right)$ reduces to

$$
\pi_{i}\left(\gamma_{i}, \gamma_{j} ; V\right)=\gamma_{j} M_{i}(V)-k_{i} .
$$

Take a given value of the outcome, $V$, in the interval $\left(A_{M}, A_{Q}\right]$. If player $j$ chooses an abandonment intensity, $\gamma_{j}$, which is strictly less than $\gamma_{j}^{*}(V)$ from Equation (42), then player $i$ 's instantaneous net benefits, $\pi_{i}\left(\gamma_{i}, \gamma_{j} ; V\right)$, is strictly negative, since $M_{i}(V)$ is positive. Hence, the best response for player $i$ would be immediate abandonment. On the other hand, if player $j$ chooses an abandonment intensity, $\gamma_{j}$, which is strictly greater than $\gamma_{j}^{*}(V)$ from Equation (42) then player $i$ 's instantaneous net benefits, $\pi_{i}\left(\gamma_{i}, \gamma_{j} ; V\right)$, is strictly positive. Hence, the best response for player $i$ would be to continue investing with zero abandonment intensity. Finally, if player $j$ chooses exactly the abandonment intensity $\gamma_{j}^{*}(V)$ from Equation (42), then player $i$ 's instantaneous net benefits, $\pi_{i}\left(\gamma_{i}, \gamma_{j} ; V\right)$, is zero for any choice of his own abandonment intensity, $\gamma_{i}$, (including immediate abandonment). Hence, player $i$ is completely indifferent between any choice of his

\footnotetext{
${ }^{25}$ The best response correspondence is a generalization of the best response function. Generally, a function returns one value for a given input. A correspondence returns a set of values for a given input. Player $i$ may have more than one best response to a given strategy from the other player (player $j$ ). The correspondence returns all these responses as a set. (Clearly player $i$ is indifferent between playing any of these strategies in the set, otherwise they would not all be part of his set of best responses.)
} 




Figure 11. The two players' best response correspondences for a given value of the outcome, $V$, in the interval $\left(A_{M}, A_{Q}\right]$. Player one's best response correspondence (the dotted line) is plotted as a function of player two's abandonment intensity, $\gamma_{2}$. That is, the independent variable is on the vertical axis and the outcome is on the horizontal axis. Player two's best response correspondence (the solid line) is plotted as a function of player one's abandonment intensity, $\gamma_{1}$. The three dots indicate the three Nash equilibria.

own abandonment intensity. To summarize, player $i$ 's the best response correspondence is

$$
\gamma_{i}^{*}\left(\gamma_{j} ; V\right)= \begin{cases}\{\infty\} & \text { when } \gamma_{j}<\gamma_{j}^{*}(V), \\ {[0, \infty]} & \text { when } \gamma_{j}=\gamma_{j}^{*}(V), \\ \{0\} & \text { when } \gamma_{j}>\gamma_{j}^{*}(V) .\end{cases}
$$

Clearly the same arguments apply to player $j$ 's best response correspondence. So player $j$ 's best response correspondence is $\gamma_{j}^{*}\left(\gamma_{i} ; V\right)$ from Equation (85).

For a given value of the outcome, $V$, in the interval $\left(A_{M}, A_{Q}\right]$, we plot the two players' best response correspondences in Figure 11. Player one's best response correspondence (the dotted line) is plotted as a function of player two's abandonment intensity, $\gamma_{2}$. That is, the independent variable $\left(\gamma_{2}\right)$ is on the vertical axis and the outcome (i.e. the set of best responses) is on the horizontal axis. Player two's best response correspondence (the solid line) is plotted as a function of player one's abandonment intensity, $\gamma_{1}$. We see that 
the two players' best response correspondences cross three times indicating three Nash equilibria. These are the two leader-follower equilibria and the variable abandonment intensity solution. Hence, we have shown that the abandonment strategies $\left(\gamma_{1}^{*}(V), \gamma_{2}^{*}(V)\right)$, from Equation (42), form a Nash equilibrium. Note the similarity between this argument and the standard arguments used to explain why a mixed strategy is a Nash equilibrium in a simple non-repeated game, (cf. Gibbons, 1992a,b, Chapter 1).

\section{Appendix F. Identical Players with Variable Abandonment Intensities: Derivation of the} abandonment intensities When $A_{Q} \geq K$.

Assume that we are in the situation when $A_{M}<K \leq \tilde{A}_{Q}$. We have already argued that $R_{i}(V)=0$ for $V \in\left(A_{M}, \tilde{A}_{Q}\right]$ and $i=1,2$. Since player $i$ is still investing in her/his investment project, $R_{i}(V)$ is still a solution to the system of ODEs

$$
\begin{aligned}
\frac{1}{2} \sigma^{2} V^{2} R_{i}^{\prime \prime}(V)+\mu V R_{i}^{\prime}(V)-\left(r+2 \lambda+\gamma_{i}(V)+\right. & \left.\gamma_{j}(V)\right) R_{i}(V) \\
& +\gamma_{j}(V) M_{i}(V)-k_{i}=0 \quad \text { when } A_{M}<V<K,
\end{aligned}
$$

and

$$
\begin{aligned}
\frac{1}{2} \sigma^{2} V^{2} R_{i}^{\prime \prime}(V)+\mu V R_{i}^{\prime}(V)-(r+2 \lambda & \left.+\gamma_{i}(V)+\gamma_{j}(V)\right) R_{i}(V) \\
& +\gamma_{j}(V) M_{i}(V)+\lambda(V-K)-k_{i}=0 \quad \text { when } K<V<\tilde{A}_{Q} .
\end{aligned}
$$

From the system of ODEs (86) we conclude that the only way that the chosen strategies for the two players can be sequentially rational is if the two players choose their abandonment strategies to be

$$
\gamma_{j}^{*}(V)= \begin{cases}\infty & \text { when } V \leq A_{M}, \\ \frac{k_{i}}{M_{i}(V)} & \text { when } A_{M}<V \leq K, \\ \frac{k_{i}-\lambda(V-K)}{M_{i}(V)} & \text { when } K<V \leq \tilde{A}_{Q}, \\ 0 & \text { when } \tilde{A}_{Q}<V .\end{cases}
$$

The argument why the abandonment strategies $\left(\gamma_{1}^{*}(V), \gamma_{2}^{*}(V)\right)$, from Equation (87), form a Nash equilibrium is the same as in the previous situation. Player $i$ 's instantaneous net benefits in this situation is

$$
\pi_{i}\left(\gamma_{i}, \gamma_{j} ; V\right)= \begin{cases}\gamma_{j} M_{i}(V)-\left(2 \lambda+\gamma_{i}+\gamma_{j}\right) R_{i}(V)-k_{i} & \text { when } A_{M}<V \leq K, \\ \lambda(V-K)+\gamma_{j} M_{i}(V)-\left(2 \lambda+\gamma_{i}+\gamma_{j}\right) R_{i}(V)-k_{i} & \text { when } K<V \leq \tilde{A}_{Q},\end{cases}
$$

for values of the outcome, $V$, in the relevant range, i.e. $V \in\left(A_{M}, \tilde{A}_{Q}\right]$. Since again the two players have the same benefits of becoming a monopolist, $M(V)$, and also have the same rate of on-going investment costs, $k$, the two players choose identical abandonment intensities. We again denote this common abandonment intensity function $\gamma^{*}(V),{ }^{26}$ and their common individual investment project value $R(V)$. For values of the outcome, $V$, above the abandonment threshold level for the follower, $\tilde{A}_{Q}$, in the corresponding leader-follower case, the value of each of the two players' investment projects is a solutions to the same ODE as we set up

\footnotetext{
${ }^{26}$ The common values of the investment projects in the corresponding monopoly model, $M(V)$, to substitute into Equation (87) is $M_{1}(V)$ from Equation (6a) when $A_{M}<V \leq K$ and $M_{2}(V)$ from Equation (6b) when $K<V \leq \tilde{A}_{Q}$.
} 
both for the follower (75) and the leader (78). Hence, the solutions to the ODE, which we denote $R_{3}(V)$, is

$$
R_{3}(V)=r_{32} V^{z_{2}}+\frac{V}{2+(r-\mu) T}-\frac{k T+K}{2+r T} \quad \text { when } \tilde{A}_{Q} \leq V .
$$

Moreover, the boundary conditions are

$$
R_{3}\left(\tilde{A}_{Q}\right)=0
$$

and

$$
R_{3}^{\prime}\left(\tilde{A}_{Q}\right)=0 .
$$

Because both the general solutions to the system of ODEs are the same as for the value of the follower's investment project, compare Equations (76) and (89), and also the boundary conditions are the same, compare Equations (77) and (90), the two players' investment projects have exactly the same value as the value of the follower's investment project in the corresponding leader-follower case. That is,

$$
R(V)=Q(V) .
$$

Finally, assume that we are in the situation when $K \leq \tilde{A}_{M}<\tilde{A}_{Q}$. We have already argued that $R_{i}(V)=0$ for $V \in\left(\tilde{A}_{M}, \tilde{A}_{Q}\right]$ and $i=1,2$. Since player $i$ is still investing in her/his investment project, $R_{i}(V)$ is still a solution to the ODE

$$
\begin{aligned}
\frac{1}{2} \sigma^{2} V^{2} R_{i}^{\prime \prime}(V)+\mu V R_{i}^{\prime}(V)-(r+2 \lambda & \left.+\gamma_{i}(V)+\gamma_{j}(V)\right) R_{i}(V) \\
& +\gamma_{j}(V) M_{i}(V)+\lambda(V-K)-k_{i}=0 \quad \text { when } \tilde{A}_{M}<V<\tilde{A}_{Q} .
\end{aligned}
$$

From the ODE (92) we conclude that the only way that the chosen strategies for the two players can be sequentially rational is if the two players choose their abandonment strategies to be

$$
\gamma_{j}^{*}(V)= \begin{cases}\infty & \text { when } V \leq \tilde{A}_{M}, \\ \frac{k_{i}-\lambda(V-K)}{M_{i}(V)} & \text { when } \tilde{A}_{M}<V \leq \tilde{A}_{Q}, \\ 0 & \text { when } \tilde{A}_{Q}<V .\end{cases}
$$

The argument why the abandonment strategies $\left(\gamma_{1}^{*}(V), \gamma_{2}^{*}(V)\right)$, from Equation (93), form a Nash equilibrium is the same as in the previous situations. Player $i$ 's instantaneous net benefits in this situation is

$$
\pi_{i}\left(\gamma_{i}, \gamma_{j} ; V\right)=\lambda(V-K)+\gamma_{j} M_{i}(V)-\left(2 \lambda+\gamma_{i}+\gamma_{j}\right) R_{i}(V)-k_{i},
$$

for values of the outcome, $V$, in the relevant range, i.e. $V \in\left(\tilde{A}_{M}, \tilde{A}_{Q}\right]$. Since again the two players have the same benefits of becoming a monopolist, $M(V)$, and also have the same rate of on-going investment costs, $k$, the two players choose identical abandonment intensities. We again denote this common abandonment intensity function $\gamma^{*}(V),{ }^{27}$ and their common individual investment project value $R(V)$. For values of the outcome, $V$, above the abandonment threshold level for the follower, $\tilde{A}_{Q}$, in the corresponding leader-follower case, the value of each of the two players' investment projects is a solutions to the same ODE as we set up

\footnotetext{
${ }^{27}$ The common value of the investment project in the corresponding monopoly model, $M(V)$, to substitute into Equation (93) is $M_{3}(V)$ from Equation (61) from Appendix A.
} 
both for the follower (75) and the leader (78). Hence, the solutions to the ODE, which we denote $R_{3}(V)$, is

$$
R_{3}(V)=r_{32} V^{z_{2}}+\frac{V}{2+(r-\mu) T}-\frac{k T+K}{2+r T} \quad \text { when } \tilde{A}_{Q} \leq V .
$$

Moreover, the boundary conditions are

$$
R_{3}\left(\tilde{A}_{Q}\right)=0
$$

and

$$
R_{3}^{\prime}\left(\tilde{A}_{Q}\right)=0 .
$$

Because both the general solutions to the system of ODEs are the same as for the value of the follower's investment project, compare Equations (76) and (95), and also the boundary conditions are the same, compare Equations (77) and (96), the two players' investment projects have exactly the same value as the value of the follower's investment project in the corresponding leader-follower case. That is,

$$
R(V)=Q(V) .
$$

\section{REFERENCES}

CARR, P. P. (1998): "Randomization and the Amercan Put," The Review of Financial Studies, 11, 597-626. Gibbons, R. (1992a): Game Theory for Applied Economists, Princeton, New Jersey, USA: Princeton University Press.

- (1992b): A Primer in Game Theory, Harlow, England: Prentice-Hall, Inc.

Hsu, J. C. And E. S. Schwartz (2006): "A Model of R\&D Valuation and the Design of Research Incentives," Working paper, The John E. Anderson Graduate School of Management at UCLA, Los Angeles, California 90095-1481, USA.

Longstaff, F. A. And E. S. Schwartz (2001): "Valuing American Options by Simulation: A Simple Least-Squares Approach," The Review of Financial Studies, 14, 113-147.

Miltersen, K. R. And E. S. Schwartz (2004): "R\&D Investments with Competitive Interactions," Review of Finance, 8, 355-401.

Pindyck, R. S. (1993): "Investments of Uncertain Cost," Journal of Financial Economics, 34, 53-76.

Schwartz, E. S. (2004): "Patents and R\&D as Real Options," Economic Notes, 33, 23-54.

Schwartz, E. S. And M. Moon (2000): "Evaluating Research and Development Investments," in Project Flexibility, Agency, and Competition, New York, New York, USA: Oxford University Press, chap. 6, 85106. 